\title{
Taste non-Goldstone, flavor-charged pseudo-Goldstone boson masses in staggered chiral perturbation theory
}

\author{
Jon A. Bailey, Hyung-Jin Kim, and Weonjong Lee \\ Lattice Gauge Theory Research Center, FPRD, and CTP, \\ Department of Physics and Astronomy, Seoul National University, Seoul, 151-747, South Korea
}

(SWME Collaboration)

(Dated: March 8, 2021)

\begin{abstract}
We calculate the masses of taste non-Goldstone pions and kaons in staggered chiral perturbation theory through next-to-leading order in the standard power counting. The results can be used to quantitatively understand taste violations in existing lattice data generated with staggered fermions and to extract the $u, d$, and $s$ quark masses and Gasser-Leutwyler parameters from the experimentally observed spectrum. The expressions for the non-Goldstone masses contain low-energy couplings unique to the non-Goldstone sector. With two exceptions these enter as coefficients of analytic terms; all the new couplings can be fixed by performing spectrum calculations. We report one-loop results for the quenched case and the fully dynamical and partially quenched $1+1+1$ and $2+1$ flavor cases in the chiral $S U(3)$ and $S U(2)$ theories.
\end{abstract}

PACS numbers: 12.38.Gc, 11.30.Rd, 12.39.Fe

Keywords: lattice QCD, staggered fermions, chiral perturbation theory, pseudo-Goldstone boson

\section{INTRODUCTION}

The masses of the up, down, and strange quarks are fundamental parameters of the Standard Model, and the low-energy couplings (LECs) of chiral perturbation theory $(\chi \mathrm{PT})$ [1, 2] parametrize the strong interactions at energies soft compared to the scale of chiral symmetry breaking, $\Lambda_{\chi}$. By fitting lattice QCD data to $\chi \mathrm{PT}$, the light quark masses, Gasser-Leutwyler couplings, and other LECs can be determined [3, 4 ].

The results of lattice QCD calculations contain discretization effects that in principle must be taken into account, either before fitting to $\chi \mathrm{PT}$ or in the fits themselves. For sufficiently small lattice spacings, lattice artifacts perturb the continuum physics [5, [6], and the effects of these perturbations at energies much less than $\Lambda_{\chi}$ can be described by an effective field theory, lattice chiral perturbation theory [7].

Staggered fermions possess an exact chiral symmetry at nonzero lattice spacing and are computationally cheap. However, in practice discretization effects known as taste violations are significant even with Symanzik improvement. In Ref. 7] Lee and Sharpe introduced the $\chi \mathrm{PT}$ for a single flavor of staggered fermion coupled to $S U(3)$ lattice gauge fields. Working to leading order (LO) in a dual expansion in the quark masses and lattice spacing, they showed that the staggered pion spectrum, including taste violations, respects $S O(4)_{T}$ taste symmetry and confirmed this prediction of the $\chi \mathrm{PT}$ by comparing to lattice data generated by using unimproved and improved versions of staggered fermions [8, 9].

Motivated by unsuccessful attempts to describe lattice data by fitting to standard continuum $\chi \mathrm{PT}$ [10], Aubin and Bernard generalized the Lee-Sharpe Lagrangian to multiple flavors to describe the effects of lattice artifacts, including taste violations, in the pseudo-Goldstone boson (PGB) sector [11 13]. They used the resulting stag- gered chiral perturbation theory $(\mathrm{S} \chi \mathrm{PT})$ to calculate the masses and decay constants of taste Goldstone pions and kaons (flavor-charged states) through one loop, including the leading chiral logarithms, which enter at next-toleading order (NLO) in the dual expansion [12, 14]. The results were used to successfully describe lattice data and factored in phenomenologically successful calculations of quark masses, meson masses, decay constants, form factors, mixing parameters, and other quantities [4, 11, 1528].

In Ref. 29] Sharpe and Van de Water enumerated the complete NLO Lagrangian of $\mathrm{S} \chi \mathrm{PT}$ and used it to predict relationships between taste breaking splittings in the PGB masses, decay constants, and dispersion relations. The NLO Lagrangian breaks $S O(4)_{T}$ to the lattice symmetry group and contributes to the masses of the PGBs terms at NLO in the dual expansion.

Lattice QCD calculations with staggered fermions are conducted by taking the fourth root of the fermion determinant to eliminate remnant doubling from the sea [4]. The conjectured relationship of the rooted staggered theory and QCD has implications that can be numerically tested. The rooting is systematically incorporated into $\mathrm{S} \chi \mathrm{PT}$ using the replica method [12, 13, 30]. Rooting leads to unphysical effects at nonzero lattice spacing. Following the arguments of Refs. 31 43], we assume that the unphysical effects of rooting vanish in the continuum limit and that $\mathrm{S} \chi \mathrm{PT}$ with the replica method correctly describes the effects of rooting that enter soft pion processes at nonzero lattice spacing.

Here we calculate the masses of the taste nonGoldstone pions and kaons through NLO (one loop) in $\mathrm{S} \chi \mathrm{PT}$. The results can be used to determine the up, down, and strange quark masses, the Gasser-Leutwyler couplings, and other quantities by confronting lattice data generated with rooted staggered fermions. Consistency between lattice data and the $\mathrm{S} \chi \mathrm{PT}$ description 
of the taste non-Goldstone sector would constitute additional numerical evidence for the conjectured relationship between the rooted staggered theory and QCD.

In Sec. III we review the formalism of $\mathrm{S} \chi \mathrm{PT}$, in Sec. III we calculate the self-energies of the taste non-Goldstone states, Sec.IV]contains the resulting one-loop corrections to the masses, and in Sec. $\mathrm{V}$ we discuss the results and note directions for future work. Appendices $\mathrm{A}, \mathrm{B}, \mathrm{C}$ and Drespectively contain a derivation of the power counting through NLO, a discussion of the taste symmetry breaking induced by NLO analytic terms, details of the loop calculations, and details of the calculation of the coefficients of the chiral logarithms.

\section{STAGGERED CHIRAL PERTURBATION THEORY}

Here we briefly review the formulation of $\mathrm{S} \chi \mathrm{PT}$ 7, 12], recalling relevant differences between the staggered theory and its continuum counterpart [1, 2]. For simplicity we consider the $4+4+4$ theory of Refs. [12, 13]. The symmetries and degrees of freedom of $\mathrm{S} \chi \mathrm{PT}$ are recalled in Sec. IA the extension of the power counting in Sec. IB and the Lagrangian in Sec. IIC

Pedagogical treatments of lattice $\chi \mathrm{PT}$ are given in Refs. [44, 45]. Investigations of the foundations of $\mathrm{S} \chi \mathrm{PT}$ were reported in Refs. [32, 34 36].

\section{A. Group theory and degrees of freedom}

In the continuum limit, the chiral symmetry of the $4+4+4$ theory is $S U(12)_{L} \times S U(12)_{R}$. Assuming spontaneous breaking to the vector subgroup, the 143 pseudoGoldstone bosons (PGBs) in the adjoint irrep of $S U(12)_{V}$ can be classified according to the continuum flavor-taste subgroup $S U(3)_{F} \times S U(4)_{T}$ :

$$
\begin{aligned}
S U(12)_{V} & \supset S U(3)_{F} \times S U(4)_{T} \\
\mathbf{1 4 3} & \rightarrow(\mathbf{8}, \mathbf{1 5}) \oplus(\mathbf{8}, \mathbf{1}) \oplus(\mathbf{1}, \mathbf{1 5})
\end{aligned}
$$

Discretization effects break the direct product of the continuum chiral symmetry and Euclidean rotations to a direct product of the lattice chiral symmetry and hypercubic rotations [12]:

$$
\begin{aligned}
& U(1)_{V} \times S U(12)_{L} \times S U(12)_{R} \times S O(4) \\
& \stackrel{a \neq 0}{\longrightarrow} U(3)_{l} \times U(3)_{r} \times\left(\Gamma_{4} \rtimes S W_{4, \text { diag }}\right)
\end{aligned}
$$

$U(3)_{l} \times U(3)_{r}$ is the lattice chiral symmetry of three flavors of staggered fermions. Its appearance ensures that a nonet of the PGBs becomes massless in the chiral limit even at nonzero lattice spacing; by definition, these are the taste Goldstone states. The $U(1)_{a}$ is not to be confused with the anomalous axial symmetry of the theory; the flavor-taste singlet meson receives a large contribution to its mass from the anomaly and is not among the PGBs.
The Clifford group $\Gamma_{4}$ is a subgroup of taste $S U(4)_{T}$; $\Gamma_{4}$ is generated by the Hermitian, $4 \times 4$ matrices $\xi_{\mu}$, $\left\{\xi_{\mu}, \xi_{\nu}\right\}=2 \delta_{\mu \nu}$ [7]. $S W_{4, \text { diag }}$ is the group of hypercubic rotations embedded in the diagonal of the direct product of Euclidean rotations and the remnant taste $S O(4)_{T}$ that emerges at energies soft compared to the scale of chiral symmetry breaking [7]:

$$
\begin{aligned}
& S O(4) \times S U(4)_{T} \stackrel{a \neq 0}{\longrightarrow} S W_{4, \operatorname{diag}} \\
& \underset{p \ll \Lambda_{\chi}}{\subset} S O(4) \times S O(4)_{T}
\end{aligned}
$$

where Eq. (6) represents the symmetry of the staggered chiral Lagrangian at leading order.

The emergence of $S O(4)_{T}$ implies degeneracies among the PGBs. The fundamental rep of $S U(4)_{T}$ is a spinor under $S O(4)_{T}$, and the $S U(4)_{T}$ adjoint and singlet of Eq. (2) fall into five irreps of $S O(4)_{T}$ [12]:

$$
\begin{aligned}
S U(4)_{T} & \supset S O(4)_{T} \\
\mathbf{1 5} & \rightarrow P \oplus A \oplus T \oplus V \\
\mathbf{1} & \rightarrow I
\end{aligned}
$$

The $S O(4)_{T}$ irreps are the pseudoscalar, axial vector, tensor, vector, and singlet (or scalar), respectively. The flavor-nonet taste-pseudoscalar PGBs are the taste Goldstone states. Among them are the pions and kaons of Refs. 12, 13]. The taste singlet $\eta^{\prime}$ receives a large contribution to its mass from the anomaly and can be integrated out of the theory.

At nonzero quark masses, the continuum chiral symmetry is softly broken to $S U(12)_{V}, S U(8)_{V} \times S U(4)_{V}$, or $S U(4)_{V} \times S U(4)_{V} \times S U(4)_{V}$ for three degenerate, two degenerate, or three non-degenerate flavors, respectively. Noting the anomaly contribution in the taste singlet sector and assuming the taste singlet PGBs are degenerate with their physical counterparts in the continuum limit, we can use the isospin, strangeness, and continuum vector symmetries to deduce the degeneracies between the remaining (taste non-singlet PGBs) and the physical states.

In doing so we consider the target continuum theory with $1+1+1$ flavors, in which there are 12 valence quarks (and 12 ghost quarks), but the fourth root has reduced the number of sea quarks from 12 flavors to three. We also restrict our attention to PGBs constructed exclusively of valence quarks. The resulting deductions from symmetry represent one of the simplest testable implications of the correctness of the rooting conjecture. We can also use them to check our $\mathrm{S} \chi \mathrm{PT}$ calculation of the masses of the taste non-Goldstone pions and kaons.

\section{B. Power counting}

The standard power counting of $\mathrm{S} \chi \mathrm{PT}[7]$ is a straightforward generalization of that in the continuum theory [2, 46]. The Lagrangian is expanded in a series of local interactions perturbing the low-energy theory about 
the chiral and continuum limits, and observables are calculated in a dual expansion in the quark masses and lattice spacing.

The order of an operator in the Lagrangian corresponds to the number of derivatives, quark mass factors, and lattice spacing factors in the operator. The symmetries of staggered fermions ensure the leading lattice artifacts are $\mathscr{O}\left(a^{2}\right)$, and derivatives always appear in pairs [7]. Let $n_{p^{2}}, n_{m}$, and $n_{a^{2}}$ be the number of derivative pairs, quark mass factors, and (squared) lattice spacing factors in an interaction. The general form of the Lagrangian is

$$
\begin{aligned}
\mathscr{L} & =\sum_{n=1}^{\infty} \mathscr{L}_{2 n}=\mathscr{L}_{2}+\mathscr{L}_{4}+\ldots \\
& =\sum_{n=1}^{\infty} \mathscr{L}_{\mathrm{N}^{n-1} \mathrm{LO}}=\mathscr{L}_{\mathrm{LO}}+\mathscr{L}_{\mathrm{NLO}}+\ldots
\end{aligned}
$$

where $n=n_{p^{2}}+n_{m}+n_{a^{2}}$ and $\mathscr{L}_{\mathrm{N}^{n-1} \mathrm{LO}} \equiv \mathscr{L}_{2 n}$.

This organization of the Lagrangian is consistent with the expectation that contributions at leading non-trivial order will be

$$
\mathscr{O}\left(p^{2} / \Lambda_{\chi}^{2}\right) \approx \mathscr{O}\left(m_{q} / \Lambda_{\chi}\right) \approx \mathscr{O}\left(a^{2} \Lambda_{\chi}^{2}\right) .
$$

This power counting is appropriate to data generated on the MILC asqtad coarse lattices $(a \approx 0.12 \mathrm{fm})$; on finer lattices or with more improved versions of the staggered action, the discretization effects are often smaller.

Feynman graphs are functions of external momenta $p_{i}$, the quark masses $m_{q}$, and the lattice spacing $a^{2}$ :

$$
\mathscr{M}\left(p_{i}, m_{q}, a^{2}\right),
$$

where the amplitude $\mathscr{M}$ is related to the $\mathbb{S}$-matrix as follows:

$$
\mathbb{S} \sim \delta^{4}\left(\sum_{i} p_{i}\right) \mathscr{M}
$$

Rescaling $p_{i}, m_{q}$, and $a^{2}$ to smaller values in accord with the power counting in Eq. (12), we have

$$
\mathscr{M}\left(p_{i}, m_{q}, a^{2}\right) \rightarrow \mathscr{M}\left(\sqrt{\varepsilon} p_{i}, \varepsilon m_{q}, \varepsilon a^{2}\right),
$$

which leads to [47]

$$
\begin{gathered}
\mathscr{M}\left(\sqrt{\varepsilon} p_{i}, \varepsilon m_{q}, \varepsilon a^{2}\right)=\varepsilon^{D} \mathscr{M}\left(p_{i}, m_{q}, a^{2}\right), \\
D=1+\sum_{n=1}^{\infty}(n-1) N_{2 n}+N_{L} .
\end{gathered}
$$

A derivation of Eq. (17) is given in Appendix A. $N_{L}$ is the number of loops in the graph, and $N_{2 n}$ is the number of vertices from operators in $\mathscr{L}_{2 n}$. From Eqs. (16) and (17), we see that loops and diagrams with vertices from higher order interactions are suppressed at small momenta, quark masses, and lattice spacings.

For any given observable we first consider all graphs with $D=1$ (LO), then those with $D=2$ (NLO), and so on. At leading order $(D=1)$ the only solutions to Eq. (17) have $N_{2 n}=0$ for $n \geq 2$ and $N_{L}=0$; i.e., only tree graphs with vertices from the LO Lagrangian are allowed. At $\mathrm{NLO}(D=2)$, the solutions have $N_{2 n}=0$ for $n \geq 3$ and either $N_{4}=1$ or $N_{L}=1$; one-loop graphs with vertices from the LO Lagrangian and tree graphs with at most one vertex from the NLO Lagrangian are allowed. In Sec. [III we use these observations to write down the graphs contributing to the PGB self-energies through NLO in the dual expansion.

\section{Lagrangian}

The Lagrangian is constructed of the PGB fields $\phi$, quark mass matrix $M$, derivatives, and taste matrices $\xi_{\mu}$ in accord with the symmetries of the terms in the effective continuum Symanzik action [7, 12, 29]. The exponential parametrization is a convenient way to include the PGBs.

$$
\Sigma=e^{i \phi / f}, \quad S U(12)_{L} \times S U(12)_{R}: \Sigma \rightarrow L \Sigma R^{\dagger}
$$

where $L, R \in S U(12)_{L, R}$ and

$$
\begin{aligned}
\phi & =\sum_{a} \phi^{a} \otimes T^{a} \\
\phi^{a} & =\left(\begin{array}{ccc}
U_{a} & \pi_{a}^{+} & K_{a}^{+} \\
\pi_{a}^{-} & D_{a} & K_{a}^{0} \\
K_{a}^{-} & \bar{K}_{a}^{0} & S_{a}
\end{array}\right) \\
T^{a} & \in\left\{\xi_{5}, i \xi_{\mu 5}, i \xi_{\mu \nu}(\mu<\nu), \xi_{\mu}, \xi_{I}\right\} .
\end{aligned}
$$

The index $a$ runs over the 16 PGB tastes in the $\mathbf{1 5}$ and $\mathbf{1}$ of $S U(4)_{T}$, the $\phi^{a}$ are Hermitian $3 \times 3$ matrices, and the $T^{a}$ are Hermitian $4 \times 4$ generators of $U(4)_{T}$, chosen (up to phases) as members of the Clifford algebra generated by the matrices $\xi_{\mu}$. With this choice for the $T^{a}$, the $S O(4)_{T}$ quantum numbers of the PGBs are explicit.

We follow Refs. 12, 29] in including the flavor-taste $S U(12)_{V}$ singlet in the Lagrangian, so $\Sigma \in U(12)$. An additional mass term in the Lagrangian accounts for the anomaly contribution to the mass of the $S U(12)_{V}$ singlet. Taking this mass correction to infinity at the end of the calculation properly decouples the $S U(12)_{V}$ singlet and yields the desired results [48, 49].

At leading order in the expansion of the Lagrangian, there are three classes of interactions: operators with $\left(n_{p^{2}}, n_{m}, n_{a^{2}}\right)=(1,0,0),(0,1,0)$, and $(0,0,1)$. We have

$$
\begin{aligned}
\mathscr{L}_{\mathrm{LO}} & =\frac{f^{2}}{8} \operatorname{Tr}\left(\partial_{\mu} \Sigma \partial_{\mu} \Sigma^{\dagger}\right)-\frac{1}{4} \mu f^{2} \operatorname{Tr}\left(M \Sigma+M \Sigma^{\dagger}\right) \\
& +\frac{2 m_{0}^{2}}{3}\left(U_{I}+D_{I}+S_{I}\right)^{2}+a^{2}\left(\mathscr{U}+\mathscr{U}^{\prime}\right)
\end{aligned}
$$

where

$$
M=\left(\begin{array}{ccc}
m_{u} & 0 & 0 \\
0 & m_{d} & 0 \\
0 & 0 & m_{s}
\end{array}\right) \otimes \xi_{I},
$$


$\xi_{I}$ is the identity matrix in taste space, and the trace (in flavor-taste space) is ordinary; we use the replica method of Damgaard and Splittorff to generalize the results of the $4+4+4$ theory to the partially quenched case [30].

The term proportional to $m_{0}^{2}$ is the contribution from the anomaly, and the potentials $\mathscr{U}$ and $\mathscr{U}^{\prime}$ break $S U(4)_{T}$ to $S O(4)_{T}$. They are

$$
\begin{aligned}
-\mathscr{U}= & C_{1} \operatorname{Tr}\left(\xi_{5}^{(n)} \Sigma \xi_{5}^{(n)} \Sigma^{\dagger}\right) \\
& +C_{6} \sum_{\mu<\nu} \operatorname{Tr}\left(\xi_{\mu \nu}^{(n)} \Sigma \xi_{\nu \mu}^{(n)} \Sigma^{\dagger}\right) \\
& +C_{3} \frac{1}{2} \sum_{\nu}\left[\operatorname{Tr}\left(\xi_{\nu}^{(n)} \Sigma \xi_{\nu}^{(n)} \Sigma\right)+h . c .\right] \\
& +C_{4} \frac{1}{2} \sum_{\nu}\left[\operatorname{Tr}\left(\xi_{\nu 5}^{(n)} \Sigma \xi_{5 \nu}^{(n)} \Sigma\right)+h . c .\right] \\
-\mathscr{U}^{\prime}= & C_{2 V} \frac{1}{4} \sum_{\nu}\left[\operatorname{Tr}\left(\xi_{\nu}^{(n)} \Sigma\right) \operatorname{Tr}\left(\xi_{\nu}^{(n)} \Sigma\right)+h . c .\right] \\
+ & C_{2 A} \frac{1}{4} \sum_{\nu}\left[\operatorname{Tr}\left(\xi_{\nu 5}^{(n)} \Sigma\right) \operatorname{Tr}\left(\xi_{5 \nu}^{(n)} \Sigma\right)+h . c .\right] \\
+ & C_{5 V} \frac{1}{2} \sum_{\nu}\left[\operatorname{Tr}\left(\xi_{\nu}^{(n)} \Sigma\right) \operatorname{Tr}\left(\xi_{\nu}^{(n)} \Sigma^{\dagger}\right)\right] \\
& +C_{5 A} \frac{1}{2} \sum_{\nu}\left[\operatorname{Tr}\left(\xi_{\nu 5}^{(n)} \Sigma\right) \operatorname{Tr}\left(\xi_{5 \nu}^{(n)} \Sigma^{\dagger}\right)\right]
\end{aligned}
$$

where $T^{a(n)}=T^{a(3)} \equiv I_{3} \otimes T^{a}$ in the $4+4+4$ theory and $T^{a}$ is given in Eq. 21] $I_{3}$ is the identity matrix in flavor space. The potentials are derived by mapping the operators of the mass dimension six effective continuum Symanzik action into the operators of $\chi \mathrm{PT}$. The remnant taste symmetry of Lee and Sharpe emerges because contributions to the potential from $S O(4)_{T}$-breaking operators in the Symanzik action are suppressed in the low-energy effective field theory by powers of the fourmomenta of the PGBs. The derivation of the potentials and the restoration of taste $S O(4)_{T}$ symmetry are described in detail in Refs. [7, 12, 29].

At NLO, the Lagrangian operators fall into six classes: $\left(n_{p^{2}}, n_{m}, n_{a^{2}}\right)=(2,0,0), \quad(0,2,0), \quad(1,1,0), \quad(1,0,1)$, $(0,1,1)$, and $(0,0,2)$. The first three contain terms analogous to those in the Gasser-Leutwyler Lagrangian [2]. The last three contain the terms enumerated by Sharpe and Van de Water [29]. The Gasser-Leutwyler terms of $\mathrm{S} \chi \mathrm{PT}$ that contribute to the PGB masses at NLO are

$$
\begin{aligned}
\mathscr{L}_{\mathrm{GL}}= & L_{4} \operatorname{Tr}\left(\partial_{\mu} \Sigma^{\dagger} \partial_{\mu} \Sigma\right) \operatorname{Tr}\left(\chi^{\dagger} \Sigma+\chi \Sigma^{\dagger}\right) \\
& +L_{5} \operatorname{Tr}\left(\partial_{\mu} \Sigma^{\dagger} \partial_{\mu} \Sigma\left(\chi^{\dagger} \Sigma+\Sigma^{\dagger} \chi\right)\right) \\
& -L_{6}\left[\operatorname{Tr}\left(\chi^{\dagger} \Sigma+\chi \Sigma^{\dagger}\right)\right]^{2} \\
& -L_{8} \operatorname{Tr}\left(\chi^{\dagger} \Sigma \chi^{\dagger} \Sigma\right)+\text { h.c. }
\end{aligned}
$$

where $\chi=2 \mu M$.

Many operators in the Sharpe-Van de Water Lagrangian contribute at NLO, but only a handful break the remnant taste $S O(4)_{T}$ to the hypercubic subgroup $S W_{4}$ of the lattice theory [29]. We use the symmetries of the Sharpe-Van de Water terms to deduce the form of their contributions to the masses; as discussed in Appendix B the explicit results of Sharpe and Van de Water for the $S O(4)_{T}$-breaking contributions to the flavorcharged PGB dispersion relations restrict the number of independent parameters in these contributions to only three.

\section{SELF-ENERGIES OF FLAVOR-CHARGED PSEUDO-GOLDSTONE BOSONS}

The symmetries protect the flavor-charged PGBs from mixing. For reasons discussed in Sec. ПI below, here we describe the calculation in the rest frame. In terms of the self-energy $\Sigma\left(p_{4}^{2}\right)$ at $\vec{p}=0$ of the state $\phi_{x y}^{a}(x \neq$ $y, x, y \in\{u, d, s\})$,

$$
M_{\phi}^{2}=m_{\phi}^{2}+\Sigma\left(-M_{\phi}^{2}\right)
$$

where $m_{\phi}$ is the tree-level (LO) mass, and $M_{\phi}$ is the (exact) mass to all orders. Noting that the perturbative expansion of $\Sigma\left(p_{4}^{2}\right)$ begins at NLO and expanding $\Sigma\left(p_{4}^{2}\right)$ in a Taylor series around $p_{4}^{2}=-m_{\phi}^{2}$ gives

$$
\begin{aligned}
M_{\phi}^{2} & =m_{\phi}^{2}+\Sigma\left(-m_{\phi}^{2}\right)-\Sigma\left(-M_{\phi}^{2}\right) \Sigma^{\prime}\left(-m_{\phi}^{2}\right)+\ldots \\
& =m_{\phi}^{2}+\Sigma\left(-m_{\phi}^{2}\right)+\mathrm{NNLO},
\end{aligned}
$$

and the NLO correction to the mass is the leading contribution to $\Sigma\left(-m_{\phi}^{2}\right)$.

In Sec. III A we consider the Feynman graphs entering the expansion of the self-energies at NLO. In Sec. III B we outline the calculation of these graphs, and in Sec. III C. we present a condensed version of the results for the $4+4+4$ theory.

\section{A. Diagrammatic expansion}

The power counting of Sec. II the Lagrangian of Sec. IIC and the definition of $\Sigma$ constrain the diagrams entering the NLO mass corrections to three types. Expanding the LO Lagrangian through $\mathscr{O}\left(\phi^{4}\right)$ and the NLO Lagrangian through $\mathscr{O}\left(\phi^{2}\right)$, we write

$$
\Sigma\left(p_{4}^{2}\right)=\frac{1}{(4 \pi f)^{2}}\left[\sigma^{c o n}\left(p_{4}^{2}\right)+\sigma^{\text {disc }}\left(p_{4}^{2}\right)\right]+\sigma^{\text {anal }}\left(p_{4}^{2}\right)+\ldots
$$

where $\sigma^{\text {con }}$ corresponds to the sum of connected tadpole diagrams (Fig. 1), $\sigma^{\text {disc }}$ corresponds to the sum of disconnected tadpoles (Fig. 2), and $\sigma^{\text {anal }}$ corresponds to the sum of tree-level diagrams (Fig. 3). The tree-level diagrams are analytic in the quark masses and (squared) lattice spacing, while the loops contribute the leading chiral logarithms.

The 4-point vertices in the tadpole graphs are from the $\mathscr{O}\left(\phi^{4}\right)$ terms in the LO Lagrangian of Eq. (22), and the 2 -point vertices in the tree-level diagrams are from the $\mathscr{O}\left(\phi^{2}\right)$ terms in the NLO Lagrangian of Eq. (26) and 


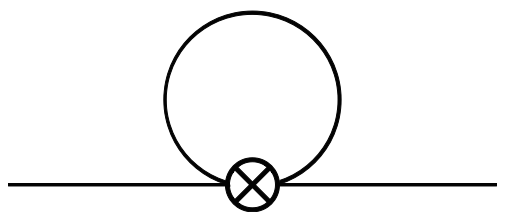

FIG. 1. At next-to-leading order, tadpole graphs contribute the leading chiral logarithms. The vertices are from the leading order Lagrangian of Eq. (22), and the propagator represents the connected part (first term) of Eq. (29).

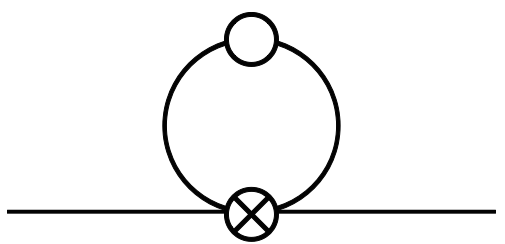

FIG. 2. Disconnected tadpoles enter in the flavor-neutral, taste-singlet, -vector, and -axial channels. The open circle represents the second term of Eq. (29).

the NLO Lagrangian of Sharpe and Van de Water 29]. The disconnected propagators (in the graphs of Fig. 2) are from quark-level disconnected contributions to the tree-level, flavor-neutral propagators in the taste singlet, axial, and vector channels [12].

The one-loop graphs break taste $S U(4)_{T}$ to the remnant taste $S O(4)_{T}$ of Ref. 7], the tree-level graphs from the Gasser-Leutwyler Lagrangian respect $S U(4)_{T}$, and the tree-level graphs from the Sharpe-Van de Water Lagrangian break $S U(4)_{T}$ in two stages: Terms of $\mathscr{O}\left(a^{2} m_{q}\right)$ and $\mathscr{O}\left(a^{4}\right)$ break $S U(4)_{T}$ to $S O(4)_{T}$, while terms of $\mathscr{O}\left(a^{2} p^{2}\right)$ break the spacetime-taste symmetry $S O(4) \times S O(4)_{T}$ to $S W_{4, \text { diag }}[29]$.

The one-loop graphs respect spacetime $S O(4)$ rotations, and the corresponding contributions to the selfenergies, $\sigma^{c o n}$ and $\sigma^{d i s c}$, are functions of $p^{2}$. The $S O(4)_{T}$-breaking analytic terms of $\mathscr{O}\left(a^{2} p^{2}\right)$, however, cannot in general be written as functions of $p^{2}$ : The dispersion relations are distorted at nonzero lattice spacing by the taste violations. To extract the masses one may consider the self-energies in the rest frame. In this case the self-energy may be written as a function of $p_{4}^{2}$, the square of the temporal component of the momentum. In Appendix $\mathrm{B}$ we recall the form of the $S O(4)_{T}$-breaking corrections to the dispersion relations [29].

\section{B. Calculation in $4+4+4$ theory}

For the $4+4+4$ theory of Ref. [12], we outline the calculation of the graphs in Figs. 11 2, and 3, After writing down the propagators and vertices (Sec. IIIB 1), we present results for the loops corresponding to each class of vertices (Sec. ЏIB 2). These intermediate results are

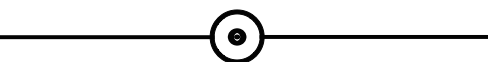

FIG. 3. At next-to-leading order, tree-level graphs contribute terms analytic in the quark masses and (squared) lattice spacing. The vertices are from the Gasser-Leutwyler and SharpeVan de Water Lagrangians of Eq. (26) and Ref. [29].

readily checked against the taste Goldstone case [12]. Sec. III contains a condensed version of these results, which can be straightforwardly generalized to the partially quenched $1+1+1$ theory and other cases of interest (Sec. IV).

We calculate the loops without extracting the vertices by summing over the flavor and taste indices in the $\mathscr{O}\left(\phi^{4}\right)$ terms in the Lagrangian. Instead we combine the expressions for the (tree-level) propagators of the flavor-charged and flavor-neutral PGBs and perform the Wick contractions before summing over the $\mathscr{O}\left(\phi^{4}\right)$ vertices. In Appendix $[\mathrm{C}$ we detail the calculation of the contributions from the mass and $a^{2} \mathscr{U}$ vertices.

\section{Propagators and vertex classes}

Expanding the LO Lagrangian of Eq. (22) through $\mathscr{O}\left(\phi^{2}\right)$ yields the propagators [12, 48]. They are

$$
\left\langle\phi_{i j}^{a} \phi_{k l}^{b}\right\rangle=\delta^{a b}\left(\delta_{i l} \delta_{j k} \frac{1}{q^{2}+\frac{1}{2}\left(I_{a}+J_{a}\right)}+\delta_{i j} \delta_{k l} D_{i l}^{a}\right)
$$

where $i, j, k, l \in\{u, d, s\}$ are flavor $S U(3)_{F}$ indices, $a, b$ are taste indices in the adjoint irrep, and

$$
\begin{aligned}
D_{i l}^{a}= & -\frac{\delta_{a}}{\left(q^{2}+I_{a}\right)\left(q^{2}+L_{a}\right)} \\
& \times \frac{\left(q^{2}+U_{a}\right)\left(q^{2}+D_{a}\right)\left(q^{2}+S_{a}\right)}{\left(q^{2}+\pi_{a}^{0}\right)\left(q^{2}+\eta_{a}\right)\left(q^{2}+\eta_{a}^{\prime}\right)},
\end{aligned}
$$

where

$$
\begin{gathered}
\delta_{I}=4 m_{0}^{2} / 3, \quad \delta_{\mu \nu}=0, \quad \delta_{5}=0 \\
\delta_{\mu}=a^{2} \delta_{V}^{\prime}, \quad \delta_{\mu 5}=a^{2} \delta_{A}^{\prime},
\end{gathered}
$$

and the names of mesons denote the squares of their treelevel masses. For $X \in\{I, J, L, U, D, S\}$,

$$
X_{a} \equiv m_{X_{a}}^{2}=2 \mu m_{x}+a^{2} \Delta_{a},
$$

where $m_{x}$ is the mass of the quark of flavor $x \in$ $\{i, j, l, u, d, s\}$, while for $X \in\left\{\pi^{0}, \eta, \eta^{\prime}\right\}$, the squares of the tree-level meson masses are the eigenvalues of the matrix

$$
\left(\begin{array}{ccc}
U_{a}+\delta_{a} & \delta_{a} & \delta_{a} \\
\delta_{a} & D_{a}+\delta_{a} & \delta_{a} \\
\delta_{a} & \delta_{a} & S_{a}+\delta_{a}
\end{array}\right)
$$


The squared tree-level mass of a flavor-charged meson $\phi_{x y}^{t}(x \neq y)$ is

$$
P_{t} \equiv \frac{1}{2}\left(X_{t}+Y_{t}\right)=\mu\left(m_{x}+m_{y}\right)+a^{2} \Delta_{t},
$$

where $X \neq Y \in\{U, D, S\}$ and $x \neq y \in\{u, d, s\}$. The hairpin couplings $\delta_{V, A}^{\prime}$ and taste splittings $\Delta_{a}$ are combinations of the couplings of the LO Lagrangian [12]:

$$
\begin{aligned}
& \delta_{V}^{\prime}=\frac{16}{f^{2}}\left(C_{2 V}-C_{5 V}\right) \\
& \delta_{A}^{\prime}=\frac{16}{f^{2}}\left(C_{2 A}-C_{5 A}\right)
\end{aligned}
$$

and

$$
\begin{aligned}
& \Delta_{I}=\frac{16}{f^{2}}\left(4 C_{3}+4 C_{4}\right) \\
& \Delta_{V}=\frac{16}{f^{2}}\left(C_{1}+3 C_{6}+C_{3}+3 C_{4}\right) \\
& \Delta_{T}=\frac{16}{f^{2}}\left(4 C_{6}+2 C_{3}+2 C_{4}\right) \\
& \Delta_{A}=\frac{16}{f^{2}}\left(C_{1}+3 C_{6}+3 C_{3}+C_{4}\right) \\
& \Delta_{P}=0 .
\end{aligned}
$$

We note the symmetry of Eqs. (36)-(42) under simultaneous interchange of vector and axial taste labels $(V \leftrightarrow A)$ and the coefficients $C_{3}$ and $C_{4}$. The axial taste matrices $i \xi_{\mu 5}$ generate the Clifford algebra; as a consequence, the LO Lagrangian is invariant under simultaneous interchange of the fields $\phi^{\mu} \leftrightarrow-\phi^{\mu 5}$ and the coefficients in the pairs $\left(C_{3}, C_{4}\right),\left(C_{2 V}, C_{2 A}\right)$, and $\left(C_{5 V}, C_{5 A}\right)$.

The minus sign arises in the unitary transformation connecting the bases $\left\{\xi_{\mu}\right\}$ and $\left\{i \xi_{\mu 5}\right\}$ : For $U$ such that

$$
\begin{aligned}
U \xi_{\mu} U^{\dagger} & =i \xi_{\mu 5}, \\
U i \xi_{\mu 5} U^{\dagger} & =-\xi_{\mu} .
\end{aligned}
$$

$U$ is unique up to a phase:

$$
U=e^{i \theta} e^{-i \frac{\pi}{4} \xi_{5}}=\frac{1}{\sqrt{2}} e^{i \theta}\left(\xi_{I}-i \xi_{5}\right)
$$

and the other taste generators are invariant under $U$.

Noting the diagrammatic expansion and the conservation of $S O(4)_{T}$, we see that taste vector and axial fields must always appear in pairs in the calculation of the selfenergies through NLO, and the minus sign in Eq. (44) is inconsequential. The results in the taste singlet, tensor, and Goldstone (pseudoscalar) channels must be invariant under interchange of the coefficients in the above pairs, while the results in the taste vector and axial channels must switch. This symmetry provides a check at each stage of the calculation.

Expanding the LO Lagrangian of Eqs. (22), (24), and (25) and keeping terms of $\mathscr{O}\left(\phi^{4}\right)$ gives 11 classes of vertices. From the kinetic energy we have two classes, from the mass terms we have one, and from the potential $a^{2} \mathscr{U}$, we have four:

$$
\begin{aligned}
\frac{f^{2}}{8} \operatorname{Tr}\left(\partial_{\mu} \Sigma \partial_{\mu} \Sigma^{\dagger}\right) & =\frac{1}{48 f^{2}} \tau_{a b c d}\left(\partial_{\mu} \phi_{i j}^{a} \phi_{j k}^{b} \partial_{\mu} \phi_{k l}^{c} \phi_{l i}^{d}-\partial_{\mu} \phi_{i j}^{a} \partial_{\mu} \phi_{j k}^{b} \phi_{k l}^{c} \phi_{l i}^{d}\right)+\ldots \\
-\frac{1}{4} \mu f^{2} \operatorname{Tr}\left(M \Sigma+M \Sigma^{\dagger}\right) & =-\frac{\mu}{48 f^{2}} \tau_{a b c d} m_{i} \phi_{i j}^{a} \phi_{j k}^{b} \phi_{k l}^{c} \phi_{l i}^{d}+\ldots \\
-a^{2} C_{1} \operatorname{Tr}\left(\xi_{5}^{(n)} \Sigma \xi_{5}^{(n)} \Sigma^{\dagger}\right) & =-\frac{a^{2} C_{1}}{12 f^{4}}\left(\tau_{a b c d}+3 \tau_{5 a b 5 c d}-4 \tau_{5 a 5 b c d}\right) \phi_{i j}^{a} \phi_{j k}^{b} \phi_{k l}^{c} \phi_{l i}^{d}+\ldots \\
-a^{2} C_{6} \sum_{\mu<\nu} \operatorname{Tr}\left(\xi_{\mu \nu}^{(n)} \Sigma \xi_{\nu \mu}^{(n)} \Sigma^{\dagger}\right) & =-\frac{a^{2} C_{6}}{12 f^{4}} \sum_{\mu<\nu}\left(\tau_{a b c d}+3 \tau_{\mu \nu, a b, \mu \nu, c d}-4 \tau_{\mu \nu, a, \mu \nu, b c d}\right) \phi_{i j}^{a} \phi_{j k}^{b} \phi_{k l}^{c} \phi_{l i}^{d}+\ldots \\
-a^{2} C_{3} \frac{1}{2} \sum_{\nu}\left[\operatorname{Tr}\left(\xi_{\nu}^{(n)} \Sigma \xi_{\nu}^{(n)} \Sigma\right)+h . c .\right] & =-\frac{a^{2} C_{3}}{12 f^{4}} \sum_{\nu}\left(\tau_{a b c d}+3 \tau_{\nu a b \nu c d}+4 \tau_{\nu a \nu b c d}\right) \phi_{i j}^{a} \phi_{j k}^{b} \phi_{k l}^{c} \phi_{l i}^{d}+\ldots \\
-a^{2} C_{4} \frac{1}{2} \sum_{\nu}\left[\operatorname{Tr}\left(\xi_{\nu 5}^{(n)} \Sigma \xi_{5 \nu}^{(n)} \Sigma\right)+h . c .\right] & =-\frac{a^{2} C_{4}}{12 f^{4}} \sum_{\nu}\left(\tau_{a b c d}+3 \tau_{\nu 5, a b, \nu 5, c d}+4 \tau_{\nu 5, a, \nu 5, b c d}\right) \phi_{i j}^{a} \phi_{j k}^{b} \phi_{k l}^{c} \phi_{l i}^{d}+\ldots
\end{aligned}
$$

where the indices $a, b, c, d$ run over the 16 tastes in the $\mathbf{1 5}$ and $\mathbf{1}$ of $S U(4)_{T}$, and $\tau_{a b c \ldots} \equiv \operatorname{Tr}\left(T^{a} T^{b} T^{c} \ldots\right)$ are traces 
of products of (Hermitian) taste matrices. The four operators of the potential $a^{2} \mathscr{U}^{\prime}$ each give one class:

$$
\begin{gathered}
-a^{2} \sum_{\nu}\left(C_{2 V} \frac{1}{4}\left[\operatorname{Tr}\left(\xi_{\nu}^{(n)} \Sigma\right) \operatorname{Tr}\left(\xi_{\nu}^{(n)} \Sigma\right)+h . c .\right]-C_{5 V} \frac{1}{2}\left[\operatorname{Tr}\left(\xi_{\nu}^{(n)} \Sigma\right) \operatorname{Tr}\left(\xi_{\nu}^{(n)} \Sigma^{\dagger}\right)\right]\right) \\
=-\frac{2 a^{2}}{3 f^{4}}\left(C_{2 V}-C_{5 V}\right) \sum_{\nu} \tau_{\nu a b c} \phi_{i i}^{\nu} \phi_{j k}^{a} \phi_{k l}^{b} \phi_{l j}^{c}+\ldots \\
-a^{2} \sum_{\nu}\left(C_{2 A} \frac{1}{4}\left[\operatorname{Tr}\left(\xi_{\nu 5}^{(n)} \Sigma\right) \operatorname{Tr}\left(\xi_{5 \nu}^{(n)} \Sigma\right)+h . c .\right]-C_{5 A} \frac{1}{2}\left[\operatorname{Tr}\left(\xi_{\nu 5}^{(n)} \Sigma\right) \operatorname{Tr}\left(\xi_{5 \nu}^{(n)} \Sigma^{\dagger}\right)\right]\right) \\
=-\frac{2 a^{2}}{3 f^{4}}\left(C_{2 A}-C_{5 A}\right) \sum_{\nu} \tau_{\nu 5, a b c} \phi_{i i}^{\nu 5} \phi_{j k}^{a} \phi_{k l}^{b} \phi_{l j}^{c}+\ldots \\
-a^{2} \sum_{\nu}\left(C_{2 V} \frac{1}{4}\left[\operatorname{Tr}\left(\xi_{\nu}^{(n)} \Sigma\right) \operatorname{Tr}\left(\xi_{\nu}^{(n)} \Sigma\right)+h . c .\right]+C_{5 V} \frac{1}{2}\left[\operatorname{Tr}\left(\xi_{\nu}^{(n)} \Sigma\right) \operatorname{Tr}\left(\xi_{\nu}^{(n)} \Sigma^{\dagger}\right)\right]\right) \\
=-\frac{a^{2}}{8 f^{4}}\left(C_{2 V}+C_{5 V}\right) \sum_{\nu} \tau_{\nu a b} \tau_{\nu c d} \phi_{i j}^{a} \phi_{j i}^{b} \phi_{k l}^{c} \phi_{l k}^{d}+\ldots \\
-a^{2} \sum_{\nu}\left(C_{2 A} \frac{1}{4}\left[\operatorname{Tr}\left(\xi_{\nu 5}^{(n)} \Sigma\right) \operatorname{Tr}\left(\xi_{5 \nu}^{(n)} \Sigma\right)+h . c .\right]+C_{5 A} \frac{1}{2}\left[\operatorname{Tr}\left(\xi_{\nu 5}^{(n)} \Sigma\right) \operatorname{Tr}\left(\xi_{5 \nu}^{(n)} \Sigma^{\dagger}\right)\right]\right) \\
=-\frac{a^{2}}{8 f^{4}}\left(C_{2 A}+C_{5 A}\right) \sum_{\nu} \tau_{\nu 5, a b} \tau_{\nu 5, c d} \phi_{i j}^{a} \phi_{j i}^{b} \phi_{k l}^{c} \phi_{l k}^{d}+\ldots
\end{gathered}
$$

Finally, expanding the Gasser-Leutwyler Lagrangian of Eq. (26) through $\mathscr{O}\left(\phi^{2}\right)$ gives

$$
\begin{aligned}
L_{4} \operatorname{Tr}\left(\partial_{\mu} \Sigma^{\dagger} \partial_{\mu} \Sigma\right) \operatorname{Tr}\left(\chi^{\dagger} \Sigma+\chi \Sigma^{\dagger}\right) & =\frac{8 L_{4}}{f^{2}} \partial_{\mu} \phi_{i j}^{a} \partial_{\mu} \phi_{j i}^{a} 4\left(U_{5}+D_{5}+S_{5}\right)+\ldots \\
L_{5} \operatorname{Tr}\left(\partial_{\mu} \Sigma^{\dagger} \partial_{\mu} \Sigma\left(\chi^{\dagger} \Sigma+\Sigma^{\dagger} \chi\right)\right) & =\frac{8 L_{5}}{f^{2}} \partial_{\mu} \phi_{i j}^{a} \partial_{\mu} \phi_{j i}^{a} I_{5}+\ldots \\
-L_{6}\left[\operatorname{Tr}\left(\chi^{\dagger} \Sigma+\chi \Sigma^{\dagger}\right)\right]^{2} & =\frac{16 L_{6}}{f^{2}} \phi_{i j}^{a} \phi_{j i}^{a} I_{5} 4\left(U_{5}+D_{5}+S_{5}\right)+\ldots \\
-L_{8} \operatorname{Tr}\left(\chi^{\dagger} \Sigma \chi^{\dagger} \Sigma\right)+\text { h.c. } & =\frac{8 L_{8}}{f^{2}} \phi_{i j}^{a} \phi_{j i}^{a} I_{5}\left(I_{5}+J_{5}\right)+\ldots
\end{aligned}
$$

The normalization of Eqs. (56) and (58) differs from that in the continuum $\chi \mathrm{PT}$ by additional factors of 4 . These factors are systematically canceled by factors of $1 / 4$ when using the replica method [12, 13, 30] to arrive at the results for the $1+1+1$ flavor theory.

\section{Results by vertex class}

We consider external fields $\phi_{x y}^{t}$ and $\phi_{y x}^{t}$, where $t$ is the taste index, $x \neq y$, and we use the renormalization scheme of Refs. 22, 12]. For the tadpole graphs with kinetic energy vertices (Eq. (460), we find

$$
\begin{aligned}
\frac{1}{12 f^{2}} \sum_{a} & {\left[p^{2}\left(\sum_{i}\left(K_{x i, i x}^{a}+K_{y i, i y}^{a}\right)-2 \theta^{a t} K_{x x, y y}^{a}\right)\right.} \\
& \left.+\sum_{i}\left(L_{x i, i x}^{a}+L_{y i, i y}^{a}\right)-2 \theta^{a t} L_{x x, y y}^{a}\right],
\end{aligned}
$$

where $i=u, d, s$ runs over the flavors in the loops, $a$ is the taste of mesons in the loops, $\theta^{a b} \equiv \frac{1}{4} \tau_{a b a b}= \pm 1$ if

$$
T^{a} T^{b} \mp T^{b} T^{a}=0, \text { and }
$$

$$
\begin{aligned}
K_{i j, k l}^{a} & \equiv \int \frac{d^{4} q}{(2 \pi)^{4}}\left\langle\phi_{i j}^{a} \phi_{k l}^{a}\right\rangle \\
L_{i j, k l}^{a} & \equiv \int \frac{d^{4} q}{(2 \pi)^{4}} q^{2}\left\langle\phi_{i j}^{a} \phi_{k l}^{a}\right\rangle .
\end{aligned}
$$

Substituting for the propagators and performing the integrals for the connected contributions gives

$$
\begin{aligned}
& \frac{1}{12 f^{2}} \sum_{a}\left[\frac{1}{(4 \pi)^{2}} \sum_{Q}\left(p^{2}-Q_{a}\right) l\left(Q_{a}\right)\right. \\
& \left.\quad+\int \frac{d^{4} q}{(2 \pi)^{4}}\left(p^{2}+q^{2}\right)\left(D_{x x}^{a}+D_{y y}^{a}-2 \theta^{a t} D_{x y}^{a}\right)\right]
\end{aligned}
$$

where $Q$ runs over the six flavor combinations $x i, y i$ for $i \in\{u, d, s\}, Q_{a}$ is the squared tree-level meson mass 
with flavor $Q$ and taste $a$, and

$$
l(X) \equiv X\left(\ln X / \Lambda^{2}+\delta_{1}(\sqrt{X} L)\right)
$$

for any squared meson mass $X$. The finite-volume correction $\delta_{1}(\sqrt{X} L)$ is [11]

$$
\delta_{1}(\sqrt{X} L) \equiv \frac{4}{\sqrt{X} L} \sum_{\vec{n} \neq \overrightarrow{0}} \frac{K_{1}(|\vec{n}| \sqrt{X} L)}{|\vec{n}|}
$$

and $\delta_{1}(\sqrt{X} L) \rightarrow 0$ in infinite volume. Here the temporal extent of the lattice is assumed infinite, $L$ is the spatial extent of the lattice, $\sqrt{X} L$ is assumed large for all values of the quark masses, $K_{1}$ is a Bessel function of imaginary argument, and the momentum in units of $2 \pi / L, \vec{n} \in \mathbb{Z}^{3}$, is summed over all integer components except $\vec{n}=\overrightarrow{0}$.

The diagrams with the mass vertices (Eq. (47)) are

$$
\begin{aligned}
\frac{1}{12 f^{2}} \sum_{a} & {\left[\sum_{i}\left[\left(m_{x y}^{2}+m_{x i}^{2}\right) K_{x i, i x}^{a}+\left(m_{x y}^{2}+m_{y i}^{2}\right) K_{y i, i y}^{a}\right]\right.} \\
& \left.+2 m_{x y}^{2} \theta^{a t} K_{x x, y y}^{a}\right]
\end{aligned}
$$

where $m_{i j}^{2}=\mu\left(m_{i}+m_{j}\right)$. Substituting for the propagators and performing the integrals for the connected diagrams gives

$$
\begin{gathered}
\frac{1}{12 f^{2}} \sum_{a}\left[\frac{1}{(4 \pi)^{2}} \sum_{Q}\left(P_{5}+Q_{5}\right) l\left(Q_{a}\right)\right. \\
+\int \frac{d^{4} q}{(2 \pi)^{4}}\left[\left(P_{5}+X_{5}\right) D_{x x}^{a}+\left(P_{5}+Y_{5}\right) D_{y y}^{a}\right. \\
\left.\left.+2 P_{5} \theta^{a t} D_{x y}^{a}\right]\right]
\end{gathered}
$$

where $X_{5}=m_{x x}^{2}$ and $Y_{5}=m_{y y}^{2}$.

For the graphs with vertices in Eqs. (48), (49), (50), and (51), from the potential $\mathscr{U}$, we obtain

$$
\frac{a^{2}}{12 f^{2}} \sum_{a}\left[\Delta_{a t} \sum_{i}\left(K_{x i, i x}^{a}+K_{y i, i y}^{a}\right)+2 \Delta_{a t}^{\prime} K_{x x, y y}^{a}\right],
$$

where

$$
\begin{aligned}
& \Delta_{a t} \equiv \frac{8}{f^{2}} \sum_{b \neq I} C_{b}\left(5+3 \theta^{a b} \theta^{b t}-4 \theta^{5 b} \theta^{b t}-4 \theta^{a b} \theta^{b 5}\right) \\
& \Delta_{a t}^{\prime} \equiv \frac{8 \theta^{a t}}{f^{2}} \sum_{b \neq I} C_{b}\left(1+3 \theta^{a b} \theta^{b t}-2 \theta^{5 b} \theta^{b t}-2 \theta^{a b} \theta^{b 5}\right)
\end{aligned}
$$

and the sum over $b$ includes all tastes appearing in the taste matrices of the vertices from $\mathscr{U}$; i.e., all tastes ex- cept the taste singlet. The coefficients $C_{b}$ are the couplings of the vertices from $\mathscr{U}$ :

$$
C_{b}= \begin{cases}C_{1} & \text { if } b=5 \\ C_{6} & \text { if } b \in\{\mu \nu\} \\ C_{3} & \text { if } b \in\{\mu\} \\ C_{4} & \text { if } b \in\{\mu 5\} .\end{cases}
$$

Substituting for the propagators and performing the integrals for the connected contributions gives

$$
\begin{aligned}
& \frac{a^{2}}{12 f^{2}} \sum_{a}\left[\frac{\Delta_{a t}}{(4 \pi)^{2}} \sum_{Q} l\left(Q_{a}\right)\right. \\
& \left.+\int \frac{d^{4} q}{(2 \pi)^{4}}\left[\Delta_{a t}\left(D_{x x}^{a}+D_{y y}^{a}\right)+2 \Delta_{a t}^{\prime} D_{x y}^{a}\right]\right] .
\end{aligned}
$$

For the contributions from the $\mathscr{U}^{\prime}$ (hairpin) vertices of Eqs. (52) and (53), we have

$$
\frac{1}{6 f^{2}} \sum_{a \in V, A} \delta_{a}\left(2+\theta^{a t}\right) \sum_{i}\left(K_{i i, x x}^{a}+K_{i i, y y}^{a}\right),
$$

where $a$ runs over the taste vector and taste axial irreps, $V=\{\mu\}$ and $A=\{\mu 5\}$. Proceeding as above, this result becomes

$$
\begin{gathered}
\frac{1}{6 f^{2}} \sum_{a \in V, A} \delta_{a}\left(2+\theta^{a t}\right)\left[\frac{1}{(4 \pi)^{2}}\left(l\left(X_{a}\right)+l\left(Y_{a}\right)\right)\right. \\
\left.+\int \frac{d^{4} q}{(2 \pi)^{4}} \sum_{i}\left[D_{i x}^{a}+D_{i y}^{a}\right]\right]
\end{gathered}
$$

where $X_{a}$ and $Y_{a}$ are given by Eq. (33). The connected and disconnected pieces of this result can be combined into a single disconnected piece by using the identity [12]

$$
\frac{1}{q^{2}+I_{a}}+\sum_{j} D_{i j}^{a}=-\frac{q^{2}+L_{a}}{\delta_{a}} D_{i l}^{a}
$$

where $I_{a}$ and $L_{a}$ are given by Eq. (33), $i, j, l \in\{u, d, s\}$, and $a \in\{V, A, I\}$. This result follows immediately from the form of $D_{i j}^{a}$ obtained by treating the $\mathscr{O}\left(\phi^{2}\right)$ terms of the LO Lagrangian that are proportional to $\delta_{a}$ as vertices and summing the resulting geometric series for the flavorneutral propagators:

$$
D_{i j}^{a}=-\frac{\delta_{a}}{\left(q^{2}+I_{a}\right)\left(q^{2}+J_{a}\right)} \frac{1}{1+\delta_{a} \sum_{l} \frac{1}{q^{2}+L_{a}}} .
$$

The equivalence of this form and that given in Eq. (30) was demonstrated in Ref. [48] for general partially quenched theories. With the use of the identity Eq. (75), Eq. (74) becomes

$$
-\frac{1}{3 f^{2}} \sum_{a \in V, A}\left(2+\theta^{a t}\right) \int \frac{d^{4} q}{(2 \pi)^{4}}\left(q^{2}+P_{a}\right) D_{x y}^{a} .
$$


For the loops from the remaining two vertices of $\mathscr{U}^{\prime}$, Eqs. (54) and (55), we find

$$
\frac{a^{2}}{32 f^{2}} \sum_{a}\left(\sum_{b \in V, A} \omega_{b} \tau_{a b t} \tau_{a b t}\left(1+\theta^{a b}\right)\right) K_{x y, y x}^{a},
$$

where

$$
\omega_{b} \equiv \frac{16}{f^{2}} \begin{cases}C_{2 V}+C_{5 V} & \text { if } b \in\{\mu\} \\ C_{2 A}+C_{5 A} & \text { if } b \in\{\mu 5\} .\end{cases}
$$

Performing the integrals gives

$$
\frac{a^{2}}{32 f^{2}} \sum_{a}\left(\sum_{b \in V, A} \omega_{b} \tau_{a b t} \tau_{a b t}\left(1+\theta^{a b}\right)\right) \frac{l\left(P_{a}\right)}{(4 \pi)^{2}} .
$$

For the tree-level graphs with the vertices in Eqs. (566), (57), (58), and (59), we have

$$
\begin{aligned}
& -\frac{16}{f^{2}}\left(2 L_{6} P_{5}+L_{4} p^{2}\right) 4\left(U_{5}+D_{5}+S_{5}\right) \\
& -\frac{16}{f^{2}}\left(2 L_{8} P_{5}+L_{5} p^{2}\right) P_{5}
\end{aligned}
$$

while the tree-level graphs with vertices from the SharpeVan de Water Lagrangian may be parametrized by introducing LECs corresponding to the irreps of $S O(4)_{T}$ and $S W_{4}$ :

$$
\begin{gathered}
-\frac{16}{f^{2}} a^{2}\left(\mathscr{A}_{t} P_{5}+\mathscr{B}_{t} 4\left(U_{5}+D_{5}+S_{5}\right)\right. \\
\left.+\mathscr{C}_{t} p_{4}^{2}+\mathscr{D}_{t} a^{2}\right),
\end{gathered}
$$

where the coefficients $\mathscr{A}_{t}, \mathscr{B}_{t}$, and $\mathscr{D}_{t}$ are degenerate within the $S O(4)_{T}$ irreps, and the coefficients $\mathscr{C}_{t}$ are degenerate within the $S W_{4}$ irreps. The symmetry of the calculation under interchange of the (valence) flavors $x \leftrightarrow y$ implies the $\mathscr{O}\left(a^{2} m_{q}\right)$ terms are proportional to $P_{5}$ or the sum $U_{5}+D_{5}+S_{5}$.

\section{Results in $4+4+4$ theory}

The results in Eqs. (63), (67), (72), (77), and (80) are the one-loop contributions to the expansion of the (negative of the) self-energies of the flavor-charged PGBs of taste $t \in\{I, \mu, \mu \nu(\mu<\nu), \mu 5,5\}$ in the $4+4+4$ theory. Collecting the connected contributions and factoring $-1 /(4 \pi f)^{2}$ gives

$$
\begin{gathered}
\sigma^{c o n}\left(p^{2}\right)=-\frac{1}{12} \sum_{a, Q}\left(p^{2}+P_{5}+a^{2} \Delta_{a t}-a^{2} \Delta_{a}\right) l\left(Q_{a}\right) \\
-\frac{a^{2}}{32} \sum_{a}\left[\sum_{b \in V, A} \omega_{b} \tau_{a b t} \tau_{a b t}\left(1+\theta^{a b}\right)\right] l\left(P_{a}\right) .
\end{gathered}
$$

where $Q$ runs over the six flavor combinations $x i$, yi for $i \in\{u, d, s\}, Q_{a}$ is the squared tree-level meson mass with flavor $Q$ and taste $a$, and $P_{a}$ is given by Eq. (35). Setting $p^{2}=-P_{t}=-P_{5}-a^{2} \Delta_{t}$, we have

$$
\begin{gathered}
\sigma^{c o n}\left(-P_{t}\right)=-\frac{1}{12} \sum_{a, Q}\left(a^{2} \Delta_{a t}-a^{2}\left(\Delta_{t}+\Delta_{a}\right)\right) l\left(Q_{a}\right) \\
-\frac{a^{2}}{32} \sum_{a}\left[\sum_{b \in V, A} \omega_{b} \tau_{a b t} \tau_{a b t}\left(1+\theta^{a b}\right)\right] l\left(P_{a}\right) .
\end{gathered}
$$

For the Goldstone case, $t=5$ and Eq. (69) with Eqs. (38) through (42) imply

$$
\Delta_{a 5}=\Delta_{a},
$$

while

$$
\tau_{a b 5} \tau_{a b 5}\left(1+\theta^{a b}\right)=0 \text { if } b \in V, A,
$$

so the connected contributions vanish identically [12].

The chiral logarithms are degenerate within the $S O(4)_{T}$ irreps; summing over the values of $a$ within each irrep, we rewrite Eq. (85):

$$
\sigma^{c o n}=-a^{2} \sum_{B}\left(\delta_{B F}^{c o n} l\left(P_{B}\right)+\frac{\Delta_{B F}^{c o n}}{12} \sum_{Q} l\left(Q_{B}\right)\right),
$$

where

$$
\begin{aligned}
\delta_{B F}^{c o n} & \equiv \frac{1}{32} \sum_{a \in B} \sum_{b \in V, A} \omega_{b} \tau_{a b t} \tau_{a b t}\left(1+\theta^{a b}\right) \\
\Delta_{B F}^{c o n} & \equiv \sum_{a \in B}\left(\Delta_{a t}-\left(\Delta_{t}+\Delta_{a}\right)\right) .
\end{aligned}
$$

The indices $B$ and $F$ designate the $S O(4)_{T}$ irreps, $B, F \in$ $\{I, V, T, A, P\}, t \in F$, and we conveniently abuse the notation to define

$$
X_{B} \equiv X_{a} \quad \text { for } a \in B \text { and } X \in\{P, Q\},
$$

which is possible because the taste splittings are degenerate within irreps of $S O(4)_{T}$.

The coefficients $\delta_{B F}^{c o n}$ and $\Delta_{B F}^{c o n}$ are linear combinations of the couplings in the potentials $\mathscr{U}^{\prime}$ and $\mathscr{U}$, respectively. Equivalently, the coefficients $\Delta_{B F}^{c o n}$ are linear combinations of the taste splittings $\Delta_{a}$, and the coefficients $\delta_{B F}^{c o n}$ are linear combinations of the couplings $\omega_{V, A}$ (defined in Eq. (79) ).

Explicit results for $\delta_{B F}^{c o n}$ and $\Delta_{B F}^{c o n}$ are given in Tables 1 and III. We note that $\delta_{P F}^{c o n}=\Delta_{P F}^{c o n}=0 . \Delta_{P F}^{c o n}=0$ follows from the symmetry of the summand of Eq. (90) under interchange of the indices $a, t$ and the vanishing of the connected contributions in the Goldstone case, $\Delta_{B P}^{c o n}=0$. The symmetry $a \leftrightarrow t$ does not exist in the summand of Eq. (89), so the relation $\delta_{P F}^{c o n}=0$ appears non-trivial. In general the symmetry $a \leftrightarrow t$ of the summand of Eq. (90) implies

$$
N_{F} \Delta_{B F}^{c o n}=N_{B} \Delta_{F B}^{c o n}
$$


TABLE I. The coefficients $\delta_{B F}^{c o n}$ defined in Eq. (89). To obtain $\delta_{B F}^{c o n}$, multiply the entry in row $B$ and column $F$ by $16 / f^{2}$.

\begin{tabular}{|c|c|c|}
\hline$\overline{B \backslash F}$ & $\overline{\bar{V}}$ & $\overline{\bar{A}}$ \\
\hline$V$ & 0 & 0 \\
\hline$A$ & 0 & 0 \\
\hline$T$ & $3\left(C_{2 A}+C_{5 A}\right)$ & $3\left(C_{2 V}+C_{5 V}\right)$ \\
\hline$P$ & 0 & 0 \\
\hline$I$ & $C_{2 V}+C_{5 V}$ & $C_{2 A}+C_{5 A}$ \\
\hline$\overline{B \backslash F}$ & $\bar{T}$ & $\bar{P}$ \\
\hline$V$ & $2\left(C_{2 A}+C_{5 A}\right)$ & \begin{tabular}{l|l}
0 & $4\left(C_{2 V}+C_{5 V}\right)$
\end{tabular} \\
\hline$A$ & $2\left(C_{2 V}+C_{5 V}\right)$ & $04\left(C_{2 A}+C_{5 A}\right)$ \\
\hline$T$ & 0 & 0 \\
\hline$P$ & 0 & 0 \\
\hline$I$ & 0 & 0 \\
\hline
\end{tabular}

where $N_{F(B)}$ is the dimension of irrep $F(B)$. Eq. (92) is useful for checking the results in Table II] Eq. (88), with the coefficients in Tables $\square$ and II is our final result for the connected tadpoles in the $4+4+4$ theory.

Collecting the disconnected pieces from the one-loop results in Eqs. (63), (67), (72), (77), and (80) gives

$$
\begin{aligned}
\sigma^{d i s c}\left(p^{2}\right)= & -\frac{(4 \pi)^{2}}{12} \sum_{a} \int \frac{d^{4} q}{(2 \pi)^{4}}\left[\left(p^{2}+P_{5}\right.\right. \\
& \left.+a^{2} \Delta_{a t}-a^{2} \Delta_{a}\right)\left(D_{x x}^{a}+D_{y y}^{a}\right) \\
& +\left[-2 \theta^{a t} p^{2}+\left(2\left(1-\theta^{a t}\right)+\rho^{a t}\right) q^{2}\right. \\
& +\left(2\left(1+\theta^{a t}\right)+\rho^{a t}\right) P_{5} \\
& \left.\left.+2 a^{2} \Delta_{a t}^{\prime}+\left(2+\rho^{a t}\right) a^{2} \Delta_{a}\right] D_{x y}^{a}\right]
\end{aligned}
$$

where

$$
\rho^{a t} \equiv \begin{cases}-4\left(2+\theta^{a t}\right) & \text { if } a \neq I \\ 0 & \text { if } a=I .\end{cases}
$$

Setting $p^{2}=-P_{t}=-P_{5}-a^{2} \Delta_{t}$, we find

$$
\begin{gathered}
\sigma^{\text {disc }}\left(-P_{t}\right)=-\frac{(4 \pi)^{2}}{12} \sum_{a} \int \frac{d^{4} q}{(2 \pi)^{4}}\left[\left(a^{2} \Delta_{a t}\right.\right. \\
\left.-a^{2}\left(\Delta_{t}+\Delta_{a}\right)\right)\left(D_{x x}^{a}+D_{y y}^{a}\right) \\
+\left[\left(2\left(1-\theta^{a t}\right)+\rho^{a t}\right) q^{2}+\left(2\left(1+2 \theta^{a t}\right)+\rho^{a t}\right) P_{5}\right. \\
\left.\left.+2 a^{2} \Delta_{a t}^{\prime}+a^{2}\left(2 \theta^{a t} \Delta_{t}+\left(2+\rho^{a t}\right) \Delta_{a}\right)\right] D_{x y}^{a}\right] .
\end{gathered}
$$

For the Goldstone case, $t=5$ and Eqs. (69), (70), and (86) imply

$$
\Delta_{a 5}^{\prime}=-\theta^{a 5} \Delta_{a}
$$

and Eq. (95) reduces to the result of Ref. [12].

The sum over a receives nonzero contributions from the $S O(4)_{T}$ vector, axial, and singlet irreps. Summing over $a$ within each irrep, we can write

$$
\begin{aligned}
& \sigma^{d i s c}=-\frac{(4 \pi)^{2}}{12} \int \frac{d^{4} q}{(2 \pi)^{4}}\left[a^{2} \Delta_{V F}^{c o n}\left(D_{x x}^{V}+D_{y y}^{V}\right)\right. \\
& +2\left(-12 P_{5}-6 q^{2} \nu_{V F}+a^{2} \Delta_{V F}^{d i s c}\right) D_{x y}^{V} \\
& +(V \rightarrow A)+a^{2} \Delta_{I F}^{c o n}\left(D_{x x}^{I}+D_{y y}^{I}\right) \\
& \left.+2\left(3 P_{5}+a^{2} \Delta_{I F}^{d i s c}\right) D_{x y}^{I}\right]
\end{aligned}
$$

where

$$
\begin{aligned}
\Delta_{B F}^{\text {disc }} & \equiv \sum_{a \in B}\left(\Delta_{a t}^{\prime}+\theta^{a t} \Delta_{t}+\left(1+\rho^{a t} / 2\right) \Delta_{a}\right) \\
\nu_{B F} & \equiv \frac{1}{2} \sum_{a \in B}\left(1+\theta^{a t}\right)
\end{aligned}
$$

for $t \in F . \nu_{B F}$ is the number of taste matrices for irrep $B \in\{V, A\}$ that commute with the taste matrix corresponding to $t \in F$. The values of $\nu_{B F}$ are given in Table IV.

The coefficients $\Delta_{B F}^{\text {disc }}$, like the coefficients $\Delta_{B F}^{c o n}$, are linear combinations of the taste splittings $\Delta_{a}$. In Appendix $\mathrm{D}$ we show that

$$
\begin{aligned}
& \Delta_{B P}^{d i s c}=0 \text { for } B=I, V, A \\
& \Delta_{V I}^{d i s c}=\Delta_{V I}^{c o n}-24 \Delta_{V} \\
& \Delta_{A I}^{d i s c}=\Delta_{A I}^{c o n}-24 \Delta_{A} .
\end{aligned}
$$

The latter two relations follow from the identity

$$
\Delta_{a t}^{\prime}=\Delta_{a t}-2\left(\Delta_{a}+\Delta_{t}\right) \quad \text { if } \theta^{a t}=1
$$

Eqs. (101) and (102) provide non-trivial checks of the

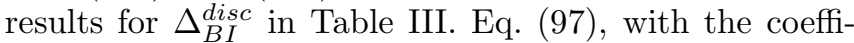
cients in Tables II, III, and IV, is our final result for the disconnected tadpoles in the $4+4+4$ theory.

Taking into account the hairpin couplings, taste splittings, and coefficients $\delta_{B F}^{c o n}, \Delta_{B F}^{c o n}$, and $\Delta_{B F}^{\text {disc }}$ in Tables $\amalg$, II] and III, we see that the loops, the results in Eqs. (88) and (97), are invariant under the symmetry

$$
\begin{aligned}
C_{3} & \leftrightarrow C_{4} \\
C_{2 V} & \leftrightarrow C_{2 A} \\
C_{5 V} & \leftrightarrow C_{5 A}
\end{aligned}
$$

or, more briefly, under

$$
V \leftrightarrow A
$$

in accord with the observations following Eq. (45) above. 
TABLE II. The coefficients $\Delta_{B F}^{c o n}$ defined in Eq. (90). To obtain $\Delta_{B F}^{c o n}$, multiply the entry in row $B$ and column $F$ by $96 / f^{2}$.

\begin{tabular}{c|c|c|c|c|c}
\hline \hline$B \backslash F$ & $V$ & $A$ & $T$ & $P$ & $I$ \\
\hline$V$ & $4 C_{1}+C_{3}+9 C_{4}+6 C_{6}$ & $4 C_{1}+3 C_{3}+3 C_{4}+6 C_{6}$ & $2 C_{3}+6 C_{4}+8 C_{6}$ & 0 & $4 C_{3}+12 C_{4}$ \\
$A$ & $4 C_{1}+3 C_{3}+3 C_{4}+6 C_{6}$ & $4 C_{1}+9 C_{3}+C_{4}+6 C_{6}$ & $6 C_{3}+2 C_{4}+8 C_{6}$ & 0 & $12 C_{3}+4 C_{4}$ \\
$T$ & $3 C_{3}+9 C_{4}+12 C_{6}$ & $9 C_{3}+3 C_{4}+12 C_{6}$ & $6 C_{3}+6 C_{4}+16 C_{6}$ & 0 & $12 C_{3}+12 C_{4}$ \\
$P$ & 0 & 0 & 0 & 0 & 0 \\
$I$ & $C_{3}+3 C_{4}$ & $3 C_{3}+C_{4}$ & $2 C_{3}+2 C_{4}$ & 0 & $4 C_{3}+4 C_{4}$ \\
\hline \hline
\end{tabular}

TABLE III. The coefficients $\Delta_{B F}^{\text {disc }}$ defined in Eq. (98). To obtain $\Delta_{B F}^{\text {disc }}$, multiply the entry in row $B$ and column $F$ by $96 / f^{2}$.

\begin{tabular}{|c|c|c|c|}
\hline$B \backslash F$ & $\bar{V}$ & & $\bar{A}$ \\
\hline$V$ & $-3 C_{1}-6 C_{4}-3 C_{6}$ & $-C$ & $1-6 C_{4}-9 C_{6}$ \\
\hline$A$ & $-C_{1}-6 C_{3}-9 C_{6}$ & $-3 C$ & $C_{1}-6 C_{3}-3 C_{6}$ \\
\hline$I$ & $C_{3}+3 C_{4}$ & & $3 C_{3}+C_{4}$ \\
\hline$\overline{B \backslash F}$ & $\bar{T}$ & $P$ & $I$ \\
\hline$V$ & $-2 C_{1}-8 C_{4}-6 C_{6}$ & 0 & $-4 C_{1}-12 C_{6}$ \\
\hline$A$ & $-2 C_{1}-8 C_{3}-6 C_{6}$ & 0 & $-4 C_{1}-12 C_{6}$ \\
\hline$I$ & $2 C_{3}+2 C_{4}$ & 0 & $4 C_{3}+4 C_{4}$ \\
\hline
\end{tabular}

TABLE IV. The numbers $\nu_{B F}$ of taste matrices for irrep $B \in$ $\{V, A\}$ that commute with any given taste matrix for irrep $F$. $\nu_{B F}$ appears in row $B$ and column $F$.

\begin{tabular}{c|c|c|c|c|c}
\hline \hline$B \backslash F$ & $V$ & $A$ & $T$ & $P$ & $I$ \\
\hline$V$ & 1 & 3 & 2 & 0 & 4 \\
$A$ & 3 & 1 & 2 & 0 & 4 \\
\hline \hline
\end{tabular}

Collecting the analytic contributions to the selfenergies from Eqs. (81), (82), and (83) gives

$$
\begin{aligned}
\sigma^{\text {anal }}\left(p_{4}^{2}\right) & =\frac{16}{f^{2}}\left(2 L_{6} P_{5}+L_{4} p_{4}^{2}\right) 4\left(U_{5}+D_{5}+S_{5}\right) \\
+ & \frac{16}{f^{2}}\left(2 L_{8} P_{5}+L_{5} p_{4}^{2}\right) P_{5} \\
+ & \frac{16}{f^{2}} a^{2}\left(\mathscr{A}_{t} P_{5}+\mathscr{B}_{t} 4\left(U_{5}+D_{5}+S_{5}\right)\right. \\
& \left.+\mathscr{C}_{t} p_{4}^{2}+\mathscr{D}_{t} a^{2}\right) .
\end{aligned}
$$

Setting $p_{4}^{2}=-P_{t}=-P_{5}-a^{2} \Delta_{t}$, we have

$$
\begin{aligned}
\sigma^{\text {anal }}= & \frac{16}{f^{2}}\left(2 L_{6}-L_{4}\right) P_{5} 4\left(U_{5}+D_{5}+S_{5}\right) \\
& +\frac{16}{f^{2}}\left(2 L_{8}-L_{5}\right) P_{5}^{2} \\
& +\frac{16}{f^{2}} a^{2}\left(\mathscr{E}_{t} P_{5}+\mathscr{F}_{t} 4\left(U_{5}+D_{5}+S_{5}\right)\right. \\
& \left.+\mathscr{G}_{t} a^{2}\right)
\end{aligned}
$$

where we have absorbed terms proportional to $a^{2} \Delta_{t}$ into the coefficients $\mathscr{E}_{t}, \mathscr{F}_{t}$, and $\mathscr{G}_{t}$. The first two lines of Eq. (109) correspond to the continuum result and are the same for all tastes. In the last two lines, the coefficients $\mathscr{F}_{t}$ are degenerate within irreps of $S O(4)_{T}$, while the coefficients $\mathscr{E}_{t}$ and $\mathscr{G}_{t}$ are degenerate within irreps of $S W_{4}$. The exact chiral symmetry implies that $\mathscr{F}_{5}=\mathscr{G}_{5}=0$. Setting $t=5$ in Eq. (109) then yields the result of Ref. [12]. In Appendix B we recall the results for the dispersion relations of Sharpe and Van de Water [29]; the $S O(4)_{T}$-breaking contributions to the $\mathscr{E}_{t}$ and $\mathscr{G}_{t}$ terms in Eq. (109) come from only three operators in the SharpeVan de Water Lagrangian. The consequences for fitting strategy are discussed in Sec. V

Eqs. (88), (97), and (109) are useful starting points for deriving results in various cases of interest. In Sec. IV we use them to deduce results for fully dynamical, partially quenched, and quenched theories.

\section{RESULTS}

The results in Eqs. (88), (97), and (109) must be modified to account for (partial) quenching [50, 51] and the fourth root of the staggered fermion determinant [4]. The replica method of Ref. [30] allows us to generalize to the partially quenched case. The replica method also allows us to systematically take into account the fourth root of the staggered determinant [13, 32, 34 36].

The effects of partial quenching and rooting in Eqs. (88), (97), and (109) are easily summarized: The valence quark masses $m_{x}$ and $m_{y}$ are no longer degenerate with the sea quark masses $m_{u}, m_{d}$, and $m_{s}$, a factor of $1 / 4$ is introduced in the second term of Eq. (88), the eigenvalues of the mass matrix in Eq. (34) are replaced with the eigenvalues of

$$
\left(\begin{array}{ccc}
U_{a}+\delta_{a} / 4 & \delta_{a} / 4 & \delta_{a} / 4 \\
\delta_{a} / 4 & D_{a}+\delta_{a} / 4 & \delta_{a} / 4 \\
\delta_{a} / 4 & \delta_{a} / 4 & S_{a}+\delta_{a} / 4
\end{array}\right)
$$

and terms in Eq. (109) that are proportional to the sum of the sea quark masses are multiplied by $1 / 4$.

Accounting for quenching the sea quarks is equally straightforward [11, 12, 50]: The second term of Eq. (88) is dropped, and the disconnected part of the propagator, 
in Eq. (30), is everywhere replaced with

$$
D_{i l}^{a, \text { quench }}=-\frac{\delta_{a}^{\text {quench }}}{\left(q^{2}+I_{a}\right)\left(q^{2}+L_{a}\right)},
$$

where

$$
\delta_{a}^{\text {quench }}= \begin{cases}4\left(m_{0}^{2}+\alpha q^{2}\right) / 3 & \text { if } a=I \\ \delta_{a} & \text { if } a \neq I .\end{cases}
$$

The one-loop contributions to the self-energies in the fully dynamical $1+1+1$ and $2+1$ flavor cases in the chiral $S U(3)$ theory are in Sec. IV A 1, One-loop contributions for partially quenched $1+1+1$ and $2+1$ flavor cases of interest are in Sec. IV A2, In Secs. IVB 1 and IVB2 we write down the analogous (fully dynamical and partially quenched) one-loop contributions in the chiral $S U(2)$ theory. Sec. IVA3 contains one-loop contributions for the quenched case.

\section{A. $S U(3)$ chiral perturbation theory}

\section{Fully dynamical case}

Introducing a factor of $1 / 4$ in the second term of Eq. (88), we obtain the connected loop contributions in the fully dynamical $1+1+1$ flavor case,

$$
\sigma^{c o n}=-a^{2} \sum_{B}\left(\delta_{B F}^{c o n} l\left(P_{B}\right)+\frac{\Delta_{B F}^{c o n}}{48} \sum_{Q} l\left(Q_{B}\right)\right),
$$

where $P_{B}$ is the LO squared mass $m_{\phi}^{2}$ of a flavor-charged PGB $\phi_{x y}^{B}$ with $\bar{x} y$ valence (anti)quarks $(x \neq y)$, and $Q_{B}$ is the LO squared mass $m_{\phi}^{2}$ of a PGB $\phi_{z \ell}^{B}$ with $\bar{z} \ell$ valence (anti)quarks, where $z \in\{x, y\}, \ell \in\{u, d, s\}$, and the sum over $Q$ runs over the six flavor combinations formed by pairing the possibilities for $z$ with those for $\ell$. Setting $x y=u d, u s, d s$ gives the results for the $\pi^{+}, K^{+}$, and $K^{0}$. We have

$$
\begin{gathered}
\sigma_{\pi^{+}}^{c o n}=-a^{2} \sum_{B}\left(\delta_{B F}^{c o n} l\left(\pi_{B}^{+}\right)+\frac{\Delta_{B F}^{c o n}}{48}\left(l\left(U_{B}\right)\right.\right. \\
\left.\left.+2 l\left(\pi_{B}^{+}\right)+l\left(K_{B}^{+}\right)+l\left(D_{B}\right)+l\left(K_{B}^{0}\right)\right)\right) \\
\sigma_{K^{+}}^{c o n}=-a^{2} \sum_{B}\left(\delta_{B F}^{c o n} l\left(K_{B}^{+}\right)+\frac{\Delta_{B F}^{c o n}}{48}\left(l\left(U_{B}\right)\right.\right. \\
\left.\left.+l\left(\pi_{B}^{+}\right)+2 l\left(K_{B}^{+}\right)+l\left(K_{B}^{0}\right)+l\left(S_{B}\right)\right)\right) \\
\sigma_{K^{0}}^{c o n}=-a^{2} \sum_{B}\left(\delta_{B F}^{c o n} l\left(K_{B}^{0}\right)+\frac{\Delta_{B F}^{c o n}}{48}\left(l\left(\pi_{B}^{+}\right)\right.\right. \\
\left.\left.+l\left(D_{B}\right)+2 l\left(K_{B}^{0}\right)+l\left(K_{B}^{+}\right)+l\left(S_{B}\right)\right)\right)
\end{gathered}
$$

where the squared tree-level masses of the flavor-charged mesons are

$$
\begin{aligned}
\pi_{B}^{+} & =\mu\left(m_{u}+m_{d}\right)+a^{2} \Delta_{B} \\
K_{B}^{+} & =\mu\left(m_{u}+m_{s}\right)+a^{2} \Delta_{B} \\
K_{B}^{0} & =\mu\left(m_{d}+m_{s}\right)+a^{2} \Delta_{B} .
\end{aligned}
$$

The integrals of the disconnected pieces, in Eq. (977), can be performed by using the residues of Ref. [12 to expand the integrands. For the $\pi^{+}$we find

$$
\begin{aligned}
& \sigma_{\pi^{+}}^{d i s c}=\frac{1}{12}\left[a ^ { 4 } \Delta _ { V F } ^ { c o n } \delta _ { V } ^ { \prime } \sum _ { X } \left(R_{U \pi^{0} \eta \eta^{\prime}}^{D S}\left(X_{V}\right) l\left(X_{V}\right)\right.\right. \\
& \left.+R_{D \pi^{0} \eta \eta^{\prime}}^{U S}\left(X_{V}\right) l\left(X_{V}\right)\right) \\
& +2 \sum_{X}\left(-12 \pi_{5}^{+}+6 X_{V} \nu_{V F}+a^{2} \Delta_{V F}^{d i s c}\right) \times \\
& \quad a^{2} \delta_{V}^{\prime} R_{\pi^{0} \eta \eta^{\prime}}^{S}\left(X_{V}\right) l\left(X_{V}\right)+(V \rightarrow A) \\
& +\frac{4}{3} a^{2} \Delta_{I F}^{c o n} \sum_{X}\left(R_{U \pi^{0} \eta}^{D S}\left(X_{I}\right) l\left(X_{I}\right)+R_{D \pi^{0} \eta}^{U S}\left(X_{I}\right) l\left(X_{I}\right)\right) \\
& \left.+\frac{8}{3}\left(3 \pi_{5}^{+}+a^{2} \Delta_{I F}^{c o n}\right) \sum_{X} R_{\pi^{0} \eta}^{S}\left(X_{I}\right) l\left(X_{I}\right)\right],
\end{aligned}
$$

where we decoupled the flavor-taste singlet, $\eta_{I}^{\prime}$, in the taste singlet channel by taking $m_{0}^{2} \rightarrow \infty$ before expanding the integrands.

In Eq. (120) we introduce a few naively perverse but extremely convenient abuses of notation. First, in each sum over $X$, the residue in the summand determines the values of the index $X$. For example, in

$$
\sum_{X} R_{U \pi^{0} \eta \eta^{\prime}}^{D S}(X) l(X)
$$

the index $X$ takes the values in the set $\left\{U, \pi^{0}, \eta, \eta^{\prime}\right\}$. When the summation over $X$ is factored from sums of residues specifying different sets of values for the index $X$, as in the first and second lines of Eq. (120), we first distribute the summation symbol and then use the residues to specify the values of $X$ in each sum. Second, the $S O(4)_{T}$ irrep specified in the argument of a given residue applies to all masses appearing in the residue. For example,

$$
R_{U \pi^{0} \eta \eta^{\prime}}^{D S}\left(U_{V}\right)=\frac{\left(D_{V}-U_{V}\right)\left(S_{V}-U_{V}\right)}{\left(\pi_{V}^{0}-U_{V}\right)\left(\eta_{V}-U_{V}\right)\left(\eta_{V}^{\prime}-U_{V}\right)},
$$

where we continue denoting squared tree-level masses by the names of the mesons. In general the residues are

$$
R_{B_{1} B_{2} \cdots B_{n}}^{A_{1} A_{2} \cdots A_{k}}\left(X_{F}\right) \equiv \frac{\prod_{A_{j}}\left(A_{j F}-X_{F}\right)}{\prod_{B_{i} \neq X}\left(B_{i F}-X_{F}\right)},
$$

where $X \in\left\{B_{1}, B_{2}, \ldots, B_{n}\right\}$ and $F \in\{V, A, I\}$ is the $S O(4)_{T}$ irrep. 
The results for the $K^{+}$and $K^{0}$ may be obtained by permuting $U, D, S$ in the residues of Eq. (120) and replacing $\pi^{+}$with $K^{+}, K^{0}$. They are

$$
\begin{aligned}
\sigma_{K^{+}}^{\text {disc }} & =\frac{1}{12} \sum_{X}\left[a ^ { 4 } \Delta _ { V F } ^ { c o n } \delta _ { V } ^ { \prime } \left(R_{U \pi^{0} \eta \eta^{\prime}}^{D S}\left(X_{V}\right) l\left(X_{V}\right)\right.\right. \\
& \left.+R_{S \pi^{0} \eta \eta^{\prime}}^{U D}\left(X_{V}\right) l\left(X_{V}\right)\right) \\
+ & 2\left(-12 K_{5}^{+}+6 X_{V} \nu_{V F}+a^{2} \Delta_{V F}^{\text {disc }}\right) \times \\
& a^{2} \delta_{V}^{\prime} R_{\pi^{0} \eta \eta^{\prime}}^{D}\left(X_{V}\right) l\left(X_{V}\right)+(V \rightarrow A) \\
+ & \frac{4}{3} a^{2} \Delta_{I F}^{c o n}\left(R_{U \pi^{0} \eta}^{D S}\left(X_{I}\right) l\left(X_{I}\right)+R_{S \pi^{0} \eta}^{U D}\left(X_{I}\right) l\left(X_{I}\right)\right) \\
+ & \left.\frac{8}{3}\left(3 K^{+}+a^{2} \Delta_{I F}^{c o n}\right) R_{\pi^{0} \eta}^{D}\left(X_{I}\right) l\left(X_{I}\right)\right]
\end{aligned}
$$

and

$$
\begin{aligned}
\sigma_{K^{0}}^{\text {disc }} & =\frac{1}{12} \sum_{X}\left[a ^ { 4 } \Delta _ { V F } ^ { c o n } \delta _ { V } ^ { \prime } \left(R_{D \pi^{0} \eta \eta^{\prime}}^{U S}\left(X_{V}\right) l\left(X_{V}\right)\right.\right. \\
& \left.+R_{S \pi^{0} \eta \eta^{\prime}}^{U D}\left(X_{V}\right) l\left(X_{V}\right)\right) \\
+ & 2\left(-12 K_{5}^{0}+6 X_{V} \nu_{V F}+a^{2} \Delta_{V F}^{d i s c}\right) \times \\
& a^{2} \delta_{V}^{\prime} R_{\pi^{0} \eta \eta^{\prime}}^{U}\left(X_{V}\right) l\left(X_{V}\right)+(V \rightarrow A) \\
+ & \frac{4}{3} a^{2} \Delta_{I F}^{c o n}\left(R_{D \pi^{0} \eta}^{U S}\left(X_{I}\right) l\left(X_{I}\right)+R_{S \pi^{0} \eta}^{U D}\left(X_{I}\right) l\left(X_{I}\right)\right) \\
+ & \left.\frac{8}{3}\left(3 K^{0}+a^{2} \Delta_{I F}^{c o n}\right) R_{\pi^{0} \eta}^{U}\left(X_{I}\right) l\left(X_{I}\right)\right] .
\end{aligned}
$$

In Eqs. (120), (124), and (125), the masses $\pi_{B}^{0}, \eta_{B}$, and $\eta_{B}^{\prime}$ are the eigenvalues of the mass matrix in Eq. (110) with $m_{u} \neq m_{d}$.

For physical values of the quark masses, strong isospin breaking is a small correction to electromagnetic isospin breaking, and $2+1$ flavor simulations have proven very useful [4]. Setting $x y=u d$, us and $m_{u}=m_{d}$ in Eq. (113) gives the connected contributions for $\pi, K$ :

$$
\begin{gathered}
\sigma_{\pi}^{c o n}=-a^{2} \sum_{B}\left(\delta_{B F}^{c o n} l\left(\pi_{B}\right)+\frac{\Delta_{B F}^{c o n}}{24}\left(2 l\left(\pi_{B}\right)+l\left(K_{B}\right)\right)\right) \\
\sigma_{K}^{c o n}=-a^{2} \sum_{B}\left(\delta_{B F}^{c o n} l\left(K_{B}\right)+\frac{\Delta_{B F}^{c o n}}{48}\left(2 l\left(\pi_{B}\right)+3 l\left(K_{B}\right)\right.\right. \\
\left.\left.+l\left(S_{B}\right)\right)\right) .
\end{gathered}
$$

In the $2+1$ flavor case, the disconnected contributions are most easily obtained by returning to Eq. (97) and performing the integrals after setting $m_{u}=m_{d}$. We find

$$
\begin{aligned}
\sigma_{\pi}^{d i s c}= & \frac{1}{12}\left[2 \sum _ { X } \left(-12 \pi_{5}+6 X_{V} \nu_{V F}\right.\right. \\
+ & \left.a^{2}\left(\Delta_{V F}^{c o n}+\Delta_{V F}^{d i s c}\right)\right) a^{2} \delta_{V}^{\prime} R_{\pi \eta \eta^{\prime}}^{S}\left(X_{V}\right) l\left(X_{V}\right) \\
+ & (V \rightarrow A) \\
+ & \left.\frac{8}{3}\left(3 \pi_{5}+2 a^{2} \Delta_{I F}^{c o n}\right)\left(\frac{3}{2} l\left(\pi_{I}\right)-\frac{1}{2} l\left(\eta_{I}\right)\right)\right] \\
\sigma_{K}^{d i s c} & =\frac{1}{12}\left[a ^ { 4 } \Delta _ { V F } ^ { c o n } \delta _ { V } ^ { \prime } \sum _ { X } \left(R_{\pi \eta \eta^{\prime}}^{S}\left(X_{V}\right) l\left(X_{V}\right)\right.\right. \\
& \left.+R_{S \eta \eta^{\prime}}^{\pi}\left(X_{V}\right) l\left(X_{V}\right)\right) \\
& +2 \sum_{X}\left(-12 K_{5}+6 X_{V} \nu_{V F}+a^{2} \Delta_{V F}^{d i s c}\right) \times \\
& a^{2} \delta_{V}^{\prime} R_{\eta \eta^{\prime}}\left(X_{V}\right) l\left(X_{V}\right)+(V \rightarrow A) \\
& +4 a^{2} \Delta_{I F}^{c o n}\left(\frac{1}{2} l\left(\pi_{I}\right)+l\left(S_{I}\right)\right) \\
& \left.+\frac{8}{3}\left(3 K_{5}-\frac{1}{4} a^{2} \Delta_{I F}^{c o n}\right) l\left(\eta_{I}\right)\right]
\end{aligned}
$$

where $\pi_{B}, \eta_{B}$, and $\eta_{B}^{\prime}(B \in\{V, A, I\})$ are the eigenvalues of the mass matrix in Eq. (110) with $m_{u}=m_{d}$, and we used the relations of the tree-level masses in the taste singlet channel to simplify the associated residues:

$$
\begin{aligned}
R_{\pi \eta}^{S}\left(\pi_{I}\right)=\frac{3}{2} & R_{\pi \eta}^{S}\left(\eta_{I}\right)=-\frac{1}{2} \\
R_{S \eta}^{\pi}\left(S_{I}\right)=3 & R_{S \eta}^{\pi}\left(\eta_{I}\right)=-2 .
\end{aligned}
$$

In the continuum limit, only the taste singlet contributions to the disconnected loops survive. Taking the continuum limits of Eqs. (126) through (129), we recover the one-loop results of Gasser and Leutwyler [2].

\section{Partially quenched case}

The connected contributions in the partially quenched $1+1+1$ flavor case have the same form as the connected contributions in the fully dynamical $1+1+1$ flavor case, Eq. (113). The difference is that the valence and sea quark masses are, in general, non-degenerate: $m_{x}, m_{y} \notin$ $\left\{m_{u}, m_{d}, m_{s}\right\}$.

For the disconnected contributions in the $1+1+1$ flavor case, keeping all quark masses in Eq. (97) distinct and 
performing the integrals as before, we find

$$
\begin{aligned}
\sigma_{x \neq y}^{d i s c}=\frac{1}{12}\left[a ^ { 4 } \Delta _ { V F } ^ { c o n } \delta _ { V } ^ { \prime } \left(R_{X \pi^{0} \eta \eta^{\prime}}^{U D S}\left(X_{V}\right) \tilde{l}\left(X_{V}\right)\right.\right. \\
+R_{Y \pi^{0} \eta \eta^{\prime}}^{U D S}\left(Y_{V}\right) \tilde{l}\left(Y_{V}\right)+\sum_{Z}\left(D_{X \pi^{0} \eta \eta^{\prime}, X}^{U D S}\left(Z_{V}\right) l\left(Z_{V}\right)\right. \\
\left.\left.+D_{Y \pi^{0} \eta \eta^{\prime}, Y}^{U D S}\left(Z_{V}\right) l\left(Z_{V}\right)\right)\right) \\
+2 \sum_{Z}\left(-12 P_{5}+6 Z_{V} \nu_{V F}+a^{2} \Delta_{V F}^{d i s c}\right) \times \\
+a^{2} \delta_{V}^{\prime} R_{X Y \pi^{0} \eta \eta^{\prime}}^{U D S}\left(Z_{V}\right) l\left(Z_{V}\right)+(V \rightarrow A) \\
+\frac{4}{3} a^{2} \Delta_{I F}^{c o n}\left(R_{X \pi^{0} \eta}^{U D S}\left(X_{I}\right) \tilde{l}\left(X_{I}\right)+R_{Y \pi^{0} \eta}^{U D S}\left(Y_{I}\right) \tilde{l}\left(Y_{I}\right)\right. \\
\left.+\sum_{Z}\left(D_{X \pi^{0} \eta, X}^{U D S}\left(Z_{I}\right) l\left(Z_{I}\right)+D_{Y \pi^{0} \eta, Y}^{U D S}\left(Z_{I}\right) l\left(Z_{I}\right)\right)\right) \\
\left.+\frac{8}{3}\left(3 P_{5}+a^{2} \Delta_{I F}^{c o n}\right) \sum_{Z} R_{X Y \pi^{0} \eta}^{U D S}\left(Z_{I}\right) l\left(Z_{I}\right)\right]
\end{aligned}
$$

where

$$
D_{B_{1} B_{2} \cdots B_{n}, B_{i}}^{A_{1} A_{2} \cdots A_{k}}\left(X_{F}\right) \equiv-\frac{\partial}{\partial B_{i F}} R_{B_{1} B_{2} \cdots B_{n}}^{A_{1} A_{2} \cdots A_{k}}\left(X_{F}\right)
$$

and

$$
\tilde{l}(X) \equiv-\left(\ln X / \Lambda^{2}+1\right)+\delta_{3}(\sqrt{X} L)
$$

The finite-volume correction $\delta_{3}(\sqrt{X} L)$ is [11]

$$
\delta_{3}(\sqrt{X} L) \equiv 2 \sum_{\vec{n} \neq \overrightarrow{0}} K_{0}(|\vec{n}| \sqrt{X} L),
$$

and $\delta_{3}(\sqrt{X} L) \rightarrow 0$ in infinite volume.

A non-trivial special case of Eq. (132) occurs for $m_{x}=$ $m_{y}$. We have

$$
\begin{aligned}
& \sigma_{x=y}^{d i s c}=\frac{1}{12}\left[2\left(-12 X_{5}+a^{2}\left(\Delta_{V F}^{c o n}+\Delta_{V F}^{d i s c}\right)\right) \times\right. \\
& a^{2} \delta_{V}^{\prime}\left(R_{X \pi^{0} \eta \eta^{\prime}}^{U D S}\left(X_{V}\right) \tilde{l}\left(X_{V}\right)+\sum_{Z} D_{X \pi^{0} \eta \eta^{\prime}, X}^{U D S}\left(Z_{V}\right) l\left(Z_{V}\right)\right) \\
& -12 a^{2} \delta_{V}^{\prime} \nu_{V F}\left(R_{X \pi^{0} \eta \eta^{\prime}}^{U D S}\left(X_{V}\right)\left(l\left(X_{V}\right)-X_{V} \tilde{l}\left(X_{V}\right)\right)\right. \\
& \left.\quad-\sum_{Z} Z_{V} D_{X \pi^{0} \eta \eta^{\prime}, X}^{U D S}\left(Z_{V}\right) l\left(Z_{V}\right)\right) \\
& +(V \rightarrow A)+\frac{8}{3}\left(3 X_{5}+2 a^{2} \Delta_{I F}^{c o n}\right)\left(R_{X \pi^{0} \eta}^{U D S}\left(X_{I}\right) \tilde{l}\left(X_{I}\right)\right. \\
& \left.\left.+\sum_{Z} D_{X \pi^{0} \eta, X}^{U D S}\left(Z_{I}\right) l\left(Z_{I}\right)\right)\right] .
\end{aligned}
$$

The masses $\pi_{B}^{0}, \eta_{B}$, and $\eta_{B}^{\prime}(B \in\{V, A, I\})$ in Eqs. (132) and (136) are the eigenvalues of the mass matrix in Eq. (110).
To obtain the connected contributions in the partially quenched $2+1$ flavor case, we set $m_{u}=m_{d}$ in Eq. (113). To obtain the disconnected contributions, we set $m_{u}=$ $m_{d}$ in Eq. (97) and consider the two cases $m_{x} \neq m_{y}$ and $m_{x}=m_{y}$ separately. We find

$$
\begin{aligned}
\sigma_{x \neq y, u=d}^{d i s c} & =\frac{1}{12}\left[a ^ { 4 } \Delta _ { V F } ^ { c o n } \delta _ { V } ^ { \prime } \left(R_{X \eta \eta^{\prime}}^{\pi S}\left(X_{V}\right) \tilde{l}\left(X_{V}\right)\right.\right. \\
+ & R_{Y \eta \eta^{\prime}}^{\pi S}\left(Y_{V}\right) \tilde{l}\left(Y_{V}\right)+\sum_{Z}\left(D_{X \eta \eta^{\prime}, X}^{\pi S}\left(Z_{V}\right) l\left(Z_{V}\right)\right. \\
+ & \left.\left.D_{Y \eta \eta^{\prime}, Y}^{\pi S}\left(Z_{V}\right) l\left(Z_{V}\right)\right)\right) \\
+ & 2 \sum_{Z}\left(-12 P_{5}+6 Z_{V} \nu_{V F}+a^{2} \Delta_{V F}^{d i s c}\right) \times \\
& a^{2} \delta_{V}^{\prime} R_{X Y \eta \eta^{\prime}}^{\pi S}\left(Z_{V}\right) l\left(Z_{V}\right)+\left(V \rightarrow A^{S}\right) \\
+ & \frac{4}{3} a^{2} \Delta_{I F}^{c o n}\left(R_{X \eta}^{\pi S}\left(X_{I}\right) \tilde{l}\left(X_{I}\right)+R_{Y \eta}^{\pi S}\left(Y_{I}\right) \tilde{l}\left(Y_{I}\right)\right. \\
+ & \left.\sum_{Z}\left(D_{X \eta, X}^{\pi S}\left(Z_{I}\right) l\left(Z_{I}\right)+D_{Y \eta, Y}^{\pi S}\left(Z_{I}\right) l\left(Z_{I}\right)\right)\right) \\
+ & \left.\frac{8}{3}\left(3 P_{5}+a^{2} \Delta_{I F}^{c o n}\right) \sum_{Z} R_{X Y \eta}^{\pi S}\left(Z_{I}\right) l\left(Z_{I}\right)\right]
\end{aligned}
$$

and

$$
\begin{aligned}
& \sigma_{x=y, u=d}^{d i s c}=\frac{1}{12}\left[2\left(-12 X_{5}+a^{2}\left(\Delta_{V F}^{c o n}+\Delta_{V F}^{d i s c}\right)\right) \times\right. \\
& a^{2} \delta_{V}^{\prime}\left(R_{X \eta \eta^{\prime}}^{\pi S}\left(X_{V}\right) \tilde{l}\left(X_{V}\right)+\sum_{Z} D_{X \eta \eta^{\prime}, X}^{\pi S}\left(Z_{V}\right) l\left(Z_{V}\right)\right) \\
& -12 a^{2} \delta_{V}^{\prime} \nu_{V F}\left(R_{X \eta \eta^{\prime}}^{\pi S}\left(X_{V}\right)\left(l\left(X_{V}\right)-X_{V} \tilde{l}\left(X_{V}\right)\right)\right. \\
& \left.\quad-\sum_{Z} Z_{V} D_{X \eta \eta^{\prime}, X}^{\pi S}\left(Z_{V}\right) l\left(Z_{V}\right)\right) \\
& \quad+(V \rightarrow A)+\frac{8}{3}\left(3 X_{5}+2 a^{2} \Delta_{I F}^{c o n}\right)\left(R_{X \eta}^{\pi S}\left(X_{I}\right) \tilde{l}\left(X_{I}\right)\right. \\
& \left.\left.\quad+\sum_{Z} D_{X \eta, X}^{\pi S}\left(Z_{I}\right) l\left(Z_{I}\right)\right)\right] .
\end{aligned}
$$

The masses $\pi_{B}, \eta_{B}$, and $\eta_{B}^{\prime}(B \in\{V, A, I\})$ appearing in Eqs. (137) and (138) are the eigenvalues of the mass matrix in Eq. (110) with $m_{u}=m_{d}$.

\section{Quenched case}

The connected loop contributions are

$$
\sigma^{c o n}=-a^{2} \sum_{B} \delta_{B F}^{c o n} l\left(P_{B}\right)
$$

To obtain the disconnected contributions, we consider Eq. (97) with the replacement $D_{i l}^{a} \rightarrow D_{i l}^{a, \text { quench }}$, where 
$D_{i l}^{a, \text { quench }}$ is given in Eqs. (111)-(112). We have

$$
\begin{aligned}
& \sigma_{x \neq y}^{d i s c}=\frac{1}{12}\left[a^{4} \Delta_{V F}^{c o n} \delta_{V}^{\prime}\left(\tilde{l}\left(X_{V}\right)+\tilde{l}\left(Y_{V}\right)\right)\right. \\
& +2\left(-12 P_{5}+a^{2} \Delta_{V F}^{d i s c}\right) a^{2} \delta_{V}^{\prime} \frac{l\left(Y_{V}\right)-l\left(X_{V}\right)}{X_{V}-Y_{V}} \\
& +12 a^{2} \delta_{V}^{\prime} \nu_{V F} \frac{Y_{V} l\left(Y_{V}\right)-X_{V} l\left(X_{V}\right)}{X_{V}-Y_{V}}+(V \rightarrow A) \\
& +\frac{4}{3} a^{2} \Delta_{I F}^{c o n}\left(\left(m_{0}^{2}-\alpha X_{I}\right) \tilde{l}\left(X_{I}\right)+\alpha l\left(X_{I}\right)\right. \\
& +(X \rightarrow Y))+\frac{8}{3}\left(3 P_{5}+a^{2} \Delta_{I F}^{c o n}\right) \times \\
& \left.\frac{\left(m_{0}^{2}-\alpha Y_{I}\right) l\left(Y_{I}\right)-\left(m_{0}^{2}-\alpha X_{I}\right) l\left(X_{I}\right)}{X_{I}-Y_{I}}\right]
\end{aligned}
$$

and

$$
\begin{aligned}
& \sigma_{x=y}^{d i s c}=\frac{1}{12}\left[2\left(-12 X_{5}+a^{2}\left(\Delta_{V F}^{c o n}+\Delta_{V F}^{d i s c}\right)\right) a^{2} \delta_{V}^{\prime} \tilde{l}\left(X_{V}\right)\right. \\
& -12 a^{2} \delta_{V}^{\prime} \nu_{V F}\left(l\left(X_{V}\right)-X_{V} \tilde{l}\left(X_{V}\right)\right) \\
& +(V \rightarrow A)+\frac{8}{3}\left(3 X_{5}+2 a^{2} \Delta_{I F}^{c o n}\right) \times \\
& \left.\left(\left(m_{0}^{2}-\alpha X_{I}\right) \tilde{l}\left(X_{I}\right)+\alpha l\left(X_{I}\right)\right)\right]
\end{aligned}
$$

where in Eq. (140) we substituted for the residues,

$$
R_{X Y}\left(X_{B}\right)=-R_{X Y}\left(Y_{B}\right)=\frac{1}{Y_{B}-X_{B}}
$$

The loop contributions to the pion and kaon masses in the case of three non-degenerate quarks and in the isospin limit can be obtained from Eqs. (139), (140), and (141) by appropriately choosing $m_{x}$ and $m_{y}$.

\section{B. $S U(2)$ chiral perturbation theory}

Expansions about the $S U(2)$ chiral limit are often better behaved than expansions about the $S U(3)$ chiral limit. The corresponding $\chi \mathrm{PT}$ was developed in Ref. [1] and extended in Refs. [52, 53] to describe results obtained with rooted staggered fermions. To date $S U(2)$ $\mathrm{S} \chi \mathrm{PT}$ analyses have been restricted to the taste Goldstone sector [53 55]. Beginning with the loop contributions from $S U(3) \mathrm{S} \chi \mathrm{PT}$, we write down corresponding $S U(2) \mathrm{S} \chi \mathrm{PT}$ loop contributions to the taste nonGoldstone PGB masses.

\section{Fully dynamical case}

From Eqs. (114), (115), and (116), we have

$$
\begin{gathered}
\sigma_{\pi^{+}}^{c o n}=-a^{2} \sum_{B}\left(\delta_{B F}^{c o n} l\left(\pi_{B}^{+}\right)+\frac{\Delta_{B F}^{c o n}}{48}\left(l\left(U_{B}\right)+2 l\left(\pi_{B}^{+}\right)\right.\right. \\
\left.\left.+l\left(D_{B}\right)\right)\right) \\
\sigma_{K^{+}}^{c o n}=-a^{2} \sum_{B} \frac{\Delta_{B F}^{c o n}}{48}\left(l\left(U_{B}\right)+l\left(\pi_{B}^{+}\right)\right) \\
\sigma_{K^{0}}^{c o n}=-a^{2} \sum_{B} \frac{\Delta_{B F}^{c o n}}{48}\left(l\left(\pi_{B}^{+}\right)+l\left(D_{B}\right)\right)
\end{gathered}
$$

To obtain the disconnected loop contributions in the $1+1+1$ flavor case, we consider Eqs. (120), (124), (125), and Eq. (97). We find

$$
\begin{aligned}
& \sigma_{\pi^{+}}^{\text {disc }}=\frac{1}{12}\left[a ^ { 4 } \Delta _ { V F } ^ { c o n } \delta _ { V } ^ { \prime } \sum _ { X } \left(R_{U \pi^{0} \eta}^{D}\left(X_{V}\right) l\left(X_{V}\right)\right.\right. \\
& \left.+R_{D \pi^{0} \eta}^{U}\left(X_{V}\right) l\left(X_{V}\right)\right) \\
& +2 \sum_{X}\left(-12 \pi_{5}^{+}+6 X_{V} \nu_{V F}+a^{2} \Delta_{V F}^{d i s c}\right) \times \\
& a^{2} \delta_{V}^{\prime} R_{\pi^{0} \eta}\left(X_{V}\right) l\left(X_{V}\right)+(V \rightarrow A) \\
& +2 a^{2} \Delta_{I F}^{c o n} \sum_{X}\left(R_{U \pi^{0}}^{D}\left(X_{I}\right) l\left(X_{I}\right)+R_{D \pi^{0}}^{U}\left(X_{I}\right) l\left(X_{I}\right)\right) \\
& \left.+4\left(3 \pi_{5}^{+}+a^{2} \Delta_{I F}^{c o n}\right) l\left(\pi_{I}^{0}\right)\right], \\
& \quad \sigma_{K^{+}}^{d i s c}=\frac{1}{12}\left[a^{4} \Delta_{V F}^{c o n} \delta_{V}^{\prime} \sum_{X} R_{U \pi^{0} \eta}^{D}\left(X_{V}\right) l\left(X_{V}\right)\right. \\
& \quad-12 \sum_{X} a^{2} \delta_{V}^{\prime} R_{\pi^{0} \eta}^{D}\left(X_{V}\right) l\left(X_{V}\right)+(V \rightarrow A) \\
& +2 a^{2} \Delta_{I F}^{c o n} \sum_{X} R_{U \pi^{0}}^{D}\left(X_{I}\right) l\left(X_{I}\right) \\
& \left.+6\left(D_{I}-\pi_{I}^{0}\right) l\left(\pi_{I}^{0}\right)\right],
\end{aligned}
$$

$$
\begin{aligned}
\sigma_{K^{0}}^{\text {disc }} & =\frac{1}{12}\left[a^{4} \Delta_{V F}^{c o n} \delta_{V}^{\prime} \sum_{X} R_{D \pi^{0} \eta}^{U}\left(X_{V}\right) l\left(X_{V}\right)\right. \\
& -12 \sum_{X} a^{2} \delta_{V}^{\prime} R_{\pi^{0} \eta}^{U}\left(X_{V}\right) l\left(X_{V}\right)+(V \rightarrow A) \\
& +2 a^{2} \Delta_{I F}^{c o n} \sum_{X} R_{D \pi^{0}}^{U}\left(X_{I}\right) l\left(X_{I}\right) \\
& \left.+6\left(U_{I}-\pi_{I}^{0}\right) l\left(\pi_{I}^{0}\right)\right]
\end{aligned}
$$


The taste vector and axial residues in Eqs. (146)-(148) can be simplified using the tree-level masses:

$$
\begin{aligned}
\pi_{B}^{0}= & \frac{1}{2}\left(U_{B}+D_{B}\right)+\frac{a^{2} \delta_{B}^{\prime}}{4} \\
& -\left(\operatorname{sgn} \delta_{B}^{\prime}\right) \frac{1}{2} \sqrt{\left(D_{B}-U_{B}\right)^{2}+\frac{1}{4}\left(a^{2} \delta_{B}^{\prime}\right)^{2}} \\
\eta_{B}= & \frac{1}{2}\left(U_{B}+D_{B}\right)+\frac{a^{2} \delta_{B}^{\prime}}{4} \\
& +\left(\operatorname{sgn} \delta_{B}^{\prime}\right) \frac{1}{2} \sqrt{\left(D_{B}-U_{B}\right)^{2}+\frac{1}{4}\left(a^{2} \delta_{B}^{\prime}\right)^{2}}
\end{aligned}
$$

for $B=V, A$. We have

$$
\begin{aligned}
R_{U \pi^{0} \eta}^{D}\left(U_{B}\right) & =\frac{4}{a^{2} \delta_{B}^{\prime}} \\
R_{U \pi^{0} \eta}^{D}\left(\pi_{B}^{0}\right) & =-\frac{2}{a^{2} \delta_{B}^{\prime}}\left(1+\sin \beta_{B}\right) \\
R_{U \pi^{0} \eta}^{D}\left(\eta_{B}\right) & =-\frac{2}{a^{2} \delta_{B}^{\prime}}\left(1-\sin \beta_{B}\right) \\
R_{D \pi^{0} \eta}^{U}\left(D_{B}\right) & =\frac{4}{a^{2} \delta_{B}^{\prime}} \\
R_{D \pi^{0} \eta}^{U}\left(\pi_{B}^{0}\right) & =-\frac{2}{a^{2} \delta_{B}^{\prime}}\left(1-\sin \beta_{B}\right) \\
R_{D \pi^{0} \eta}^{U}\left(\eta_{B}\right) & =-\frac{2}{a^{2} \delta_{B}^{\prime}}\left(1+\sin \beta_{B}\right) \\
R_{\pi^{0} \eta}\left(\pi_{B}^{0}\right) & =\frac{2}{a^{2} \delta_{B}^{\prime}} \cos \beta_{B} \\
R_{\pi^{0} \eta}\left(\eta_{B}\right) & =-\frac{2}{a^{2} \delta_{B}^{\prime}} \cos \beta_{B} \\
R_{\pi^{0} \eta}^{D}\left(\pi_{B}^{0}\right) & =\frac{1}{2}\left(1+\sin \beta_{B}-\cos \beta_{B}\right) \\
R_{\pi^{0} \eta}^{D}\left(\eta_{B}\right) & =\frac{1}{2}\left(1-\sin \beta_{B}+\cos \beta_{B}\right),
\end{aligned}
$$

where

$$
\begin{aligned}
& \sin \beta_{B} \equiv\left(\operatorname{sgn} \delta_{B}^{\prime}\right) \frac{D_{B}-U_{B}}{\sqrt{\left(D_{B}-U_{B}\right)^{2}+\frac{1}{4}\left(a^{2} \delta_{B}^{\prime}\right)^{2}}} \\
& \cos \beta_{B} \equiv\left(\operatorname{sgn} \delta_{B}^{\prime}\right) \frac{\frac{1}{2} a^{2} \delta_{B}^{\prime}}{\sqrt{\left(D_{B}-U_{B}\right)^{2}+\frac{1}{4}\left(a^{2} \delta_{B}^{\prime}\right)^{2}}} .
\end{aligned}
$$

In the isospin limit, $\beta_{B}=0$.

The connected loops in the $2+1$ flavor case are

$$
\begin{aligned}
\sigma_{\pi}^{c o n} & =-a^{2} \sum_{B}\left(\delta_{B F}^{c o n}+\frac{\Delta_{B F}^{c o n}}{12}\right) l\left(\pi_{B}\right) \\
\sigma_{K}^{c o n} & =-a^{2} \sum_{B} \frac{\Delta_{B F}^{c o n}}{24} l\left(\pi_{B}\right),
\end{aligned}
$$

and the disconnected loops are

$$
\begin{aligned}
\sigma_{\pi}^{d i s c}=\frac{1}{12}[ & 4\left(-12 \pi_{5}+a^{2}\left(\Delta_{V F}^{c o n}+\Delta_{V F}^{d i s c}\right)\right) \times \\
& \left(l\left(\pi_{V}\right)-l\left(\eta_{V}\right)\right) \\
+ & 24 \nu_{V F}\left(\pi_{V} l\left(\pi_{V}\right)-\eta_{V} l\left(\eta_{V}\right)\right) \\
+ & (V \rightarrow A) \\
+ & \left.4\left(3 \pi_{5}+2 a^{2} \Delta_{I F}^{c o n}\right) l\left(\pi_{I}\right)\right]
\end{aligned}
$$

$$
\begin{gathered}
\sigma_{K}^{d i s c}=\frac{1}{12}\left[2 a^{2} \Delta_{V F}^{c o n}\left(l\left(\pi_{V}\right)-l\left(\eta_{V}\right)\right)-12 a^{2} \delta_{V}^{\prime} l\left(\eta_{V}\right)\right. \\
\left.+(V \rightarrow A)+2 a^{2} \Delta_{I F}^{c o n} l\left(\pi_{I}\right)\right] .
\end{gathered}
$$

The masses in Eqs. (163), (164), (165), and (166) are

$$
\begin{aligned}
& \pi_{B}=2 \mu m_{u}+a^{2} \Delta_{B} \quad \forall B \\
& \eta_{B}=2 \mu m_{u}+a^{2} \Delta_{B}+\frac{a^{2} \delta_{B}^{\prime}}{2} \quad B \in\{V, A\} .
\end{aligned}
$$

All mesons circulating in loops in the $S U(2)$ chiral theory are pions.

\section{Partially quenched case}

We obtain the connected contributions in the $1+1+1$ flavor case by dropping terms in Eq. (113) corresponding to loops with a strange sea quark; i.e., the sum over $Q$ excludes the $x s$ and $y s$ mesons, and we treat the $x$ and $y$ quarks as light 1 To obtain the disconnected contributions in the $1+1+1$ flavor case, we consider Eqs. (132),

\footnotetext{
1 Another case of interest would be that of a single heavy (strange) valence quark, $m_{y} \sim m_{s} \gg m_{x} \sim m_{u, d}$.
} 
(136), and (97). We have

$$
\begin{aligned}
\sigma_{x \neq y}^{d i s c}=\frac{1}{12}\left[a ^ { 4 } \Delta _ { V F } ^ { c o n } \delta _ { V } ^ { \prime } \left(R_{X \pi^{0} \eta}^{U D}\left(X_{V}\right) \tilde{l}\left(X_{V}\right)\right.\right. \\
+R_{Y \pi^{0} \eta}^{U D}\left(Y_{V}\right) \tilde{l}\left(Y_{V}\right)+\sum_{Z}\left(D_{X \pi^{0} \eta, X}^{U D}\left(Z_{V}\right) l\left(Z_{V}\right)\right. \\
\left.\left.+D_{Y \pi^{0} \eta, Y}^{U D}\left(Z_{V}\right) l\left(Z_{V}\right)\right)\right) \\
+2 \sum_{Z}\left(-12 P_{5}+6 Z_{V} \nu_{V F}+a^{2} \Delta_{V F}^{d i s c}\right) \times \\
\quad a^{2} \delta_{V}^{\prime} R_{X Y \pi^{0} \eta}^{U D}\left(Z_{V}\right) l\left(Z_{V}\right)+(V \rightarrow A) \\
+2 a^{2} \Delta_{I F}^{c o n}\left(R_{X \pi^{0}}^{U D}\left(X_{I}\right) \tilde{l}\left(X_{I}\right)+R_{Y \pi^{0}}^{U D}\left(Y_{I}\right) \tilde{l}\left(Y_{I}\right)\right. \\
\left.+\sum_{Z}\left(D_{X \pi^{0}, X}^{U D}\left(Z_{I}\right) l\left(Z_{I}\right)+D_{Y \pi^{0}, Y}^{U D}\left(Z_{I}\right) l\left(Z_{I}\right)\right)\right) \\
\left.+4\left(3 P_{5}+a^{2} \Delta_{I F}^{c o n}\right) \sum_{Z} R_{X Y \pi^{0}}^{U D}\left(Z_{I}\right) l\left(Z_{I}\right)\right]
\end{aligned}
$$

and

$$
\begin{aligned}
& \sigma_{x=y}^{d i s c}=\frac{1}{12}\left[2\left(-12 X_{5}+a^{2}\left(\Delta_{V F}^{c o n}+\Delta_{V F}^{d i s c}\right)\right) \times\right. \\
& a^{2} \delta_{V}^{\prime}\left(R_{X \pi^{0} \eta}^{U D}\left(X_{V}\right) \tilde{l}\left(X_{V}\right)+\sum_{Z} D_{X \pi^{0} \eta, X}^{U D}\left(Z_{V}\right) l\left(Z_{V}\right)\right) \\
& \quad-12 a^{2} \delta_{V}^{\prime} \nu_{V F}\left(R_{X \pi^{0} \eta}^{U D}\left(X_{V}\right)\left(l\left(X_{V}\right)-X_{V} \tilde{l}\left(X_{V}\right)\right)\right. \\
& \left.-\sum_{Z} Z_{V} D_{X \pi^{0} \eta, X}^{U D}\left(Z_{V}\right) l\left(Z_{V}\right)\right) \\
& +(V \rightarrow A)+4\left(3 X_{5}+2 a^{2} \Delta_{I F}^{c o n}\right)\left(R_{X \pi^{0}}^{U D}\left(X_{I}\right) \tilde{l}\left(X_{I}\right)\right. \\
& \left.\left.+\sum_{Z} D_{X \pi^{0}, X}^{U D}\left(Z_{I}\right) l\left(Z_{I}\right)\right)\right]
\end{aligned}
$$

Setting $m_{u}=m_{d}$, we have the disconnected contributions in the $2+1$ flavor case:

$$
\begin{aligned}
& \sigma_{x \neq y, u=d}^{d i s c}=\frac{1}{12}\left[a ^ { 4 } \Delta _ { V F } ^ { c o n } \delta _ { V } ^ { \prime } \left(R_{X \eta}^{\pi}\left(X_{V}\right) \tilde{l}\left(X_{V}\right)\right.\right. \\
& +R_{Y \eta}^{\pi}\left(Y_{V}\right) \tilde{l}\left(Y_{V}\right)+\sum_{Z}\left(D_{X \eta, X}^{\pi}\left(Z_{V}\right) l\left(Z_{V}\right)\right. \\
& \left.\left.+D_{Y \eta, Y}^{\pi}\left(Z_{V}\right) l\left(Z_{V}\right)\right)\right) \\
& +2 \sum_{Z}\left(-12 P_{5}+6 Z_{V} \nu_{V F}+a^{2} \Delta_{V F}^{d i s c}\right) \times \\
& \quad a^{2} \delta_{V}^{\prime} R_{X Y \eta}^{\pi}\left(Z_{V}\right) l\left(Z_{V}\right)+\left(V \rightarrow A^{\prime}\right) \\
& +2 a^{2} \Delta_{I F}^{c o n}\left(\left(l\left(X_{I}\right)+\left(\pi_{I}-X_{I}\right) \tilde{l}\left(X_{I}\right)\right)\right. \\
& \left.+\left(l\left(Y_{I}\right)+\left(\pi_{I}-Y_{I}\right) \tilde{l}\left(Y_{I}\right)\right)\right) \\
& \left.+4\left(3 P_{5}+a^{2} \Delta_{I F}^{c o n}\right) \sum_{Z} R_{X Y}^{\pi}\left(Z_{I}\right) l\left(Z_{I}\right)\right]
\end{aligned}
$$

and

$$
\begin{aligned}
& \sigma_{x=y, u=d}^{d i s c}=\frac{1}{12}\left[2\left(-12 X_{5}+a^{2}\left(\Delta_{V F}^{c o n}+\Delta_{V F}^{\text {disc }}\right)\right) \times\right. \\
& a^{2} \delta_{V}^{\prime}\left(R_{X \eta}^{\pi}\left(X_{V}\right) \tilde{l}\left(X_{V}\right)+\sum_{Z} D_{X \eta, X}^{\pi}\left(Z_{V}\right) l\left(Z_{V}\right)\right) \\
& -12 a^{2} \delta_{V}^{\prime} \nu_{V F}\left(R_{X \eta}^{\pi}\left(X_{V}\right)\left(l\left(X_{V}\right)-X_{V} \tilde{l}\left(X_{V}\right)\right)\right. \\
& \left.-\sum_{Z} Z_{V} D_{X \eta, X}^{\pi}\left(Z_{V}\right) l\left(Z_{V}\right)\right)+(V \rightarrow A) \\
& \left.+4\left(3 X_{5}+2 a^{2} \Delta_{I F}^{c o n}\right)\left(l\left(X_{I}\right)+\left(\pi_{I}-X_{I}\right) \tilde{l}\left(X_{I}\right)\right)\right]
\end{aligned}
$$

\section{CONCLUSION}

Our final results for the masses of the flavor-charged PGBs through NLO in $\mathrm{S} \chi \mathrm{PT}$ are given by adding Eq. (28) evaluated on-shell to the tree-level (LO) result of Eq. (35). These results and others of interest can be obtained from those in the $4+4+4$ flavor theory given in Eqs. (88), (97), and (109) of Sec. (IIIC). Applying the replica method to reduce the number of tastes per flavor from four to one gives the connected tadpole, disconnected tadpole, and NLO (analytic) tree-level contributions to the on-shell self-energies. In Sec. IV we write down the connected and disconnected tadpoles in the $1+1+1$ flavor and $2+1$ flavor cases in $S U(3)$ and $S U(2)$ $\chi \mathrm{PT}$.

For the fully dynamical case with three non-degenerate quarks, the results in the $S U(3)$ chiral theory are in 
Eqs. (114) through (125). The corresponding results in the isospin limit are in Eqs. (126) through (129). Expansions about the $S U(2)$ chiral limit are given in Eqs. (143) through (148) and Eqs. (163) through (166). For the quenched case, the results are in Eqs. (139) through (141), where the LECs are the quenched counterparts of those in the theories with dynamical quarks.

For the partially quenched case, the connected contributions have the same form as those in the fully dynamical case, Eq. (113). For three non-degenerate sea quarks, the disconnected contributions in the $S U(3)$ chiral theory are in Eqs. (132) and (136). Taking the isospin limit in the sea, the corresponding results are in Eqs. (137) and (138). The expansions about the $S U(2)$ chiral limit are given in Eqs. (169)-(170) and Eqs. (171)-(172).

The LO contributions to the masses break taste $S U(4)_{T}$ to taste $S O(4)_{T}$ [7]. At NLO the (tadpole) loops respect taste $S O(4)_{T}$, the tree-level counterterms from the Gasser-Leutwyler Lagrangian respect taste $S U(4)_{T}$, and tree-level counterterms from the Sharpe-Van de Water Lagrangian break spacetime-taste $S O(4) \times S U(4)_{T}$ to the lattice symmetry, $\Gamma_{4} \rtimes S W_{4, \text { diag }}$.

The pattern of taste symmetry breaking is illustrated in Fig. 4. Regarded as functions of the valence masses, the LO masses receive corrections at NLO to their slopes and intercepts. The chiral logarithms contribute to both types of corrections but do not lift degeneracies within taste $S O(4)_{T}$ irreps. A small subset of the Sharpe-Van de Water counterterms breaks the $S O(4)_{T}$ symmetry. With HYP-smeared staggered valence quarks on MILC coarse lattices, the corrections to the intercepts are smaller than the statistical uncertainties [56, 57]; Fig. 44represents this case. The exact chiral symmetry at nonzero lattice spacing ensures corrections to the intercept of the taste Goldstone $(P)$ mesons vanish.

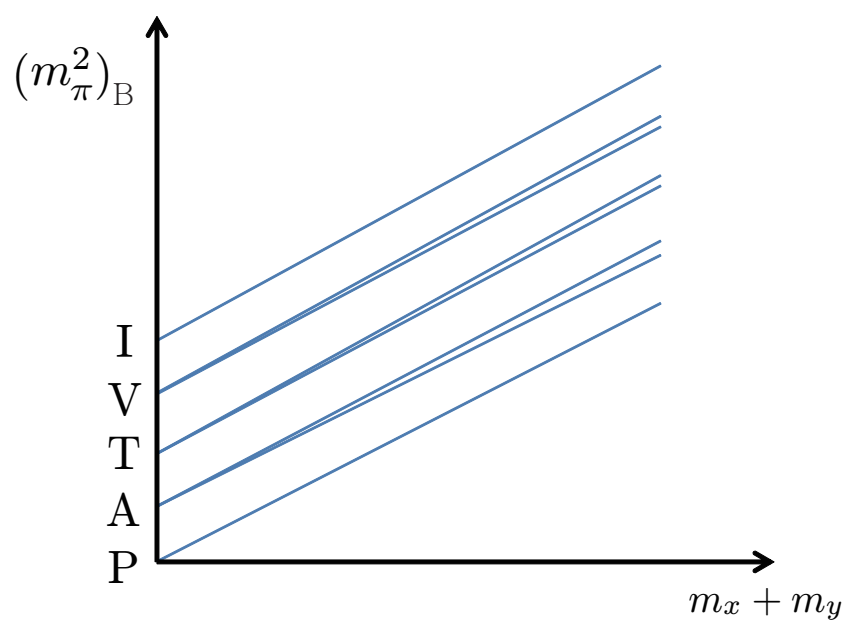

FIG. 4. Pattern of taste symmetry breaking in squared PGB masses. The PGBs fall into 8 lattice irreps, and the masses receive corrections to the slopes and intercepts that lift the degeneracy of the taste $S O(4)_{T}$ irreps. The corrections to the intercepts are of $\mathscr{O}\left(a^{4}\right)$ and very small on typical lattices.
As discussed in Appendix $\mathrm{B}$ the $S O(4)_{T}$-breaking contributions to the masses of the flavor-charged PGBs arise from only three operators in the Sharpe-Van de Water Lagrangian [29]. As a direct consequence, the $S O(4)_{T^{-}}$ breaking corrections to the slopes and intercepts of Fig. 4 depend on only three LECs. One could obtain them from the splittings in the slopes of the taste vector, axial, and tensor irreps.

The LECs of the LO taste-breaking potentials enter the results in four specific ways: The couplings of the potential $\mathscr{U}$, Eq. (24), enter only via the tree-level mass splittings and the coefficients of the chiral logarithms from disconnected tadpoles; for each taste channel, these coefficients are given in Tables [I] and [II. The couplings of the potential $\mathscr{U}^{\prime}$, Eq. (25), enter only via the hairpin coefficients of the Goldstone sector and the two linear combinations of Eq. (79). The former arise in disconnected propagators, while the latter multiply connected tadpoles with valence-valence mesons in the loop; for each taste channel, they are given in Table \.

From Eqs. (38)-(42) and Tables II and III we observe that the coefficients of the chiral logarithms from the disconnected tadpoles are completely determined by the tree-level mass splittings. The tree-level mass splittings also determine the coefficients of chiral logarithms from connected tadpoles with sea quarks in the loop. Having determined the $S O(4)_{T}$-breaking terms and the LO masses, one could perform fits to partially quenched data to extract the remaining (two) coefficients of the connected contributions and the coefficients of the $S O(4)_{T^{-}}$ preserving analytic corrections at NLO. We note in passing that the coefficients of the quenched and $S U(2)$ chiral theories are different from the coefficients of the $S U(3)$ chiral theory.

The calculation here can be extended to mixed action $\chi \mathrm{PT}$ and to other quantities of phenomenological interest such as decay constants, form factors, and mixing parameters. For example, one could consider HISQ or HYP-smeared staggered on asqtad staggered simulations; the $\mathrm{S} \chi \mathrm{PT}$ for both cases is the same [58 61]. A calculation for $B_{K}$ is given in Ref. [27]. We plan to calculate in the near future the one-loop corrections to the mass spectrum of pions and kaons in the mixed action case.

\section{ACKNOWLEDGMENTS}

This research was supported by the Creative Research Initiatives program (3348-20090015) of the NRF grant of the Korean government (MEST). We thank Steve Sharpe for hospitality during our visit at the University of Washington. We thank Steve Sharpe and Claude Bernard for helpful discussions. 


\section{Appendix A: Power counting formula}

In Sec. IIB we recalled the standard power counting of $\mathrm{S} \chi \mathrm{PT}$. Here we derive the power counting formula, Eq. (17). The derivation is based closely on the discussion for the continuum case in Ref. [47].

We begin by noting that the effective continuum Symanzik action contains no operators of mass dimension five or seven [29]:

$$
S_{\mathrm{SYM}}=S_{4}+a^{2} S_{6}+a^{4} S_{8}+\ldots
$$

For the following discussion, we assume without proof that no operators of odd mass dimension appear at higher orders in the Symanzik action. At the end of the derivation, we consider the restrictions this assumption places on the validity of Eq. (17).

Mapping the operators of the Symanzik action into the Lagrangian of $\mathrm{S} \chi \mathrm{PT}$ and using it to compute an arbitrary amplitude, we note that dependence on the lattice spacing enters via the vertices and the (tree-level) propagators. By the assumption of the previous paragraph, all vertices and propagators depend analytically on $a^{2}$. The Symanzik and S $\chi \mathrm{PT}$ actions are translation invariant, so momentum conservation holds in $\mathrm{S} \chi \mathrm{PT}$, as in the continuum theory.

The lattice-spacing dependence of the propagators can be deduced from Eqs. (29), (30), (32), (33), (35), (34), and Eqs. (36) through (42). The propagators receive corrections proportional to the hairpin couplings of Eqs. (36) and (37) and the taste splittings of Eqs. (38) through (42). The former enter only the disconnected parts of the flavor-neutral propagators; the latter are corrections to the tree-level masses of all the PGBs.

In any given amplitude, internal lines contribute factors of

$$
\begin{gathered}
\int \frac{d^{4} q}{(2 \pi)^{4}}\left\langle\phi_{i j}^{a} \phi_{k l}^{b}\right\rangle \\
=\delta^{a b} \int \frac{d^{4} q}{(2 \pi)^{4}}\left(\delta_{i l} \delta_{j k} \frac{1}{q^{2}+\frac{1}{2}\left(I_{a}+J_{a}\right)}+\delta_{i j} \delta_{k l} D_{i l}^{a}\right),
\end{gathered}
$$

and vertices contribute momentum-conserving delta functions and couplings of the form

$$
v=p^{2 n_{p^{2}}-n_{q}} q^{n_{q}} m_{q}^{n_{m}} a^{2 n_{a^{2}}},
$$

where $2 n_{p^{2}}$ is the number of derivatives in the interaction, $p$ is an external momentum, $q$ is an internal momentum, $n_{q}$ is the number of internal lines contracted with the vertex, $n_{m}$ is the number of quark-mass factors from the vertex, $m_{q}$ is a light $(u, d$, or $s)$ quark mass, $2 n_{a^{2}}$ is the number of lattice-spacing factors from the vertex, and $a$ is the lattice spacing.

Rescaling the external momenta, quark masses, and lattice spacing,

$$
\begin{aligned}
p & \rightarrow \sqrt{\varepsilon} \\
m_{q} & \rightarrow \varepsilon m_{q} \\
a^{2} & \rightarrow \varepsilon a^{2},
\end{aligned}
$$

the factors from internal lines scale as

$$
\int \frac{d^{4} q}{(2 \pi)^{4}}\left\langle\phi_{i j}^{a} \phi_{k l}^{b}\right\rangle \rightarrow \varepsilon \int \frac{d^{4} q}{(2 \pi)^{4}}\left\langle\phi_{i j}^{a} \phi_{k l}^{b}\right\rangle,
$$

where we changed the variable of integration from $q$ to $\sqrt{\varepsilon} q$. Under the same change of variable and the rescaling of Eq. (A5), the momentum-conserving delta functions scale as

$$
\begin{aligned}
\delta^{4}(p+q) & \rightarrow \delta^{4}(\sqrt{\varepsilon}(p+q)) \\
& =\frac{1}{\varepsilon^{2}} \delta^{4}(p+q),
\end{aligned}
$$

while the vertex contribution of Eq. (A44) scales as

$$
\begin{aligned}
v & \rightarrow \varepsilon^{n_{p^{2}}+n_{m}+n_{a^{2}} v} \\
& =\varepsilon^{n} v,
\end{aligned}
$$

where $n \equiv n_{p^{2}}+n_{m}+n_{a^{2}}$. Recalling the definitions of $n_{p^{2}}, n_{m}$, and $n_{a^{2}}$ and the organization of the Lagrangian in Eq. (10), we conclude that Eq. (A12) implies that a vertex contribution scales with a factor of $\varepsilon^{n}$ if and only if the vertex is from an interaction in the Lagrangian $\mathscr{L}_{2 n}$ of Eq. (10).

A given Feynman graph $\mathscr{M}\left(p_{i}, m_{q}, a^{2}\right)$ has $N_{I}$ internal lines and $N_{V}$ vertices, where

$$
N_{V}=\sum_{n=1}^{\infty} N_{2 n}
$$

i.e., the number of vertices in the graph is the sum of the number of vertices $N_{2 n}$ from each term $\mathscr{L}_{2 n}$ in the Lagrangian. Because the total momentum flowing into the diagram equals the total momentum flowing out, one momentum-conserving delta function does not contribute an independent constraint and is factored out of the amplitude. Multiplying the rescaling factors of the internal lines, vertex contributions, and remaining $N_{V}-1$ momentum-conserving delta functions gives

$$
\mathscr{M}\left(p_{i}, m_{q}, a^{2}\right) \rightarrow \varepsilon^{D} \mathscr{M}\left(p_{i}, m_{q}, a^{2}\right)
$$

where

$$
D=N_{I}-2\left(N_{V}-1\right)+\sum_{n=1}^{\infty} n N_{2 n} .
$$

The number of loops in a diagram is the number of independent integrations after imposing the $N_{V}-1$ constraints from the momentum-conserving delta functions:

$$
N_{L}=N_{I}-\left(N_{V}-1\right) \text {. }
$$


Using this relation to eliminate $N_{I}$ from Eq. A15 gives the desired result:

$$
\begin{aligned}
D & =N_{L}-N_{V}+1+\sum_{n=1}^{\infty} n N_{2 n} \\
& =1+N_{L}+\sum_{n=1}^{\infty}(n-1) N_{2 n} .
\end{aligned}
$$

We now reflect on the validity of this result. We assumed that no operators of odd mass dimension appear at any order in the Symanzik action. This assumption is known to be true only through mass dimension eight. In principle it could be violated at mass dimension nine:

$$
S_{\mathrm{SYM}}=S_{4}+a^{2} S_{6}+a^{4} S_{8}+a^{5} S_{9}+\ldots
$$

In this case the rescaling is the same for the internal lines and the momentum-conserving delta functions, but the possible vertex contributions are different:

$$
v=p^{n_{p}-n_{q}} q^{n_{q}} m_{q}^{n_{m}} a^{n_{a}},
$$

and the expansion of the $\mathrm{S} \chi \mathrm{PT}$ Lagrangian can be written

$$
\mathscr{L}=\sum_{n=2,4,5, \ldots} \mathscr{L}_{n}
$$

We allow for the number of derivatives $n_{p}$ in an operator to be odd because they are the only objects in the chiral Lagrangian with indices that can contract with those of taste matrices to construct operators with an odd number of taste spurions.

The vertex factors now rescale as

$$
v \rightarrow \varepsilon^{n / 2} v,
$$

where $n \equiv n_{p}+2 n_{m}+n_{a}$, and we have

$$
N_{V}=\sum_{n} N_{n}
$$

The modified power counting formula is

$$
D=1+N_{L}+\sum_{n=2,4,5, \ldots}\left(\frac{n}{2}-1\right) N_{n},
$$

and writing out the solutions to this equation for $D=$ $1,2, \frac{5}{2}, 3$ yields the same solutions to the power counting relation for $D=1,2,3$ as before. For $D=1,2$, the new operators in $\mathscr{L}_{5}$ do not contribute, and the power counting of Eq. (17) is justified through NLO.

\section{Appendix B: NLO analytic corrections and taste symmetry breaking}

The NLO analytic corrections to the PGB masses break spacetime-taste $S O(4) \times S O(4)_{T}$ to the diagonal hypercubic subgroup $S W_{4, \text { diag }}$ of the lattice theory. Here we consider the responsible operators and note the pattern of symmetry breaking in the mass spectrum.

Sharpe and Van de Water enumerated the NLO Lagrangian giving rise to the NLO analytic corrections [29]. Although many operators contribute at NLO, the vast majority respect the remnant taste symmetry $S O(4)_{T}$; only three operators are responsible for the symmetry breaking in the masses of the flavor-charged PGBs, and all are of type $\left(n_{p^{2}}, n_{m}, n_{a^{2}}\right)=(1,0,1)$. For example, the operator

$$
\frac{a^{2} C_{36 V}}{f^{2}} \sum_{\mu} \operatorname{Tr}\left(\partial_{\mu} \phi \xi_{\mu} \partial_{\mu} \phi \xi_{\mu}\right)
$$

yields the correction to the self-energy for $\phi_{x y}^{t}$

$$
\frac{8 a^{2} C_{36 V}}{f^{2}} \sum_{\mu} p_{\mu} p_{\mu} \theta^{\mu t},
$$

which in the dispersion relations breaks (spatial) rotation invariance and lifts the $S O(4)_{T}$ degeneracies of the masses of the PGBs.

The symmetry breaking corrections to the dispersion relations were calculated by Sharpe and Van de Water. They have the form

$$
\begin{aligned}
E_{I}^{2} & =\vec{p}^{2}+M_{I}^{2}\left(1+\kappa_{I}\right) \\
E_{4}^{2} & =\vec{p}^{2}\left(1+\kappa_{4}-\kappa_{i}\right)+M_{V}^{2}\left(1+\kappa_{4}\right) \\
E_{i}^{2} & =p_{i}^{2}\left(1+\kappa_{i}-\kappa_{4}\right)+p_{j}^{2}+p_{k}^{2}+M_{V}^{2}\left(1+\kappa_{i}\right) \\
E_{i j}^{2} & =\left(p_{i}^{2}+p_{j}^{2}\right)\left(1+\kappa_{i j}-\kappa_{i 4}\right)+p_{k}^{2}+M_{T}^{2}\left(1+\kappa_{i j}\right) \\
E_{i 4}^{2} & =p_{i}^{2}+\left(p_{j}^{2}+p_{k}^{2}\right)\left(1+\kappa_{i 4}-\kappa_{i j}\right)+M_{T}^{2}\left(1+\kappa_{i 4}\right) \\
E_{i 5}^{2} & =p_{i}^{2}\left(1+\kappa_{i 5}-\kappa_{45}\right)+p_{j}^{2}+p_{k}^{2}+M_{A}^{2}\left(1+\kappa_{i 5}\right) \\
E_{45}^{2} & =\vec{p}^{2}\left(1+\kappa_{45}-\kappa_{i 5}\right)+M_{A}^{2}\left(1+\kappa_{45}\right) \\
E_{5}^{2} & =\vec{p}^{2}+M_{P}^{2}\left(1+\kappa_{5}\right),
\end{aligned}
$$

where $M_{t}$ is the flavor-charged PGB mass through NLO including all but the taste $S O(4)_{T}$ violating contributions, and we use $\kappa_{t}$ to denote the $\delta_{t}$ of Ref. [29]. Writing out the various contributions to $M_{t}$ more explicitly,

$$
M_{t}^{2}=m_{x y}^{2}+a^{2} \Delta_{t}+\ell_{t}+a_{t},
$$

where the first two terms are the LO result, $\ell_{t}$ is the sum of all loop corrections for taste $t$, and $a_{t}$ is the sum of all NLO analytic corrections respecting taste $S O(4)_{T}$. The results of Sharpe and Van de Water then imply that the NLO $S O(4)_{T}$-breaking corrections are

$$
\left(m_{x y}^{2}+a^{2} \Delta_{t}\right) \kappa_{t}=m_{x y}^{2} \kappa_{t}+a^{2} \Delta_{t} \kappa_{t} .
$$

Since the corrections $\kappa_{t}$ are proportional to $a^{2}$, the NLO masses of the flavor-charged PGBs, considered as functions of $m_{x}+m_{y}$, receive corrections to their slopes and 
intercepts. However, these corrections are completely determined by only three a priori unknown LECs in the Sharpe-Van de Water Lagrangian.

Recalling the $S O(4)_{T}$-breaking corrections in Eq. (109),

$$
\Xi_{t}=\frac{16 a^{2}}{f^{2}}\left(m_{x y}^{2} \mathscr{E}_{t}+a^{2} \mathscr{G}_{t}\right)
$$

we see that $\Xi_{t}$ contains the 16 a priori independent coefficients $\mathscr{E}_{t}$ and $\mathscr{G}_{t}$, and that the part of $\Xi_{t}$ which breaks $S O(4)_{T}$ is fixed by only three LECs in the Sharpe-Van de Water Lagrangian. The remaining part, which is $S O(4)_{T}$-symmetric, can be determined by fitting as discussed in Sec. V.

In summary, the $S O(4)_{T}$-breaking corrections to the dispersion relations imply the presence of $\mathscr{O}\left(a^{2} m_{q}\right)$ and $\mathscr{O}\left(a^{4}\right)$ analytic corrections to the masses of the corresponding (flavor-charged) PGBs. These corrections are determined by only three LECs, which may be taken to be the splittings of the $S O(4)_{T}$ vector, tensor, and axial irreps.

\section{Appendix C: Example loop calculations}

In Sec. III B 2 we wrote down the results for each class of vertices in the graphs contributing to the PGB selfenergies at NLO. Here we detail the calculation of these results in two cases: for the (mass) vertices of Eq. (47), which yield Eq. (66), and the $\left(a^{2} \mathscr{U}\right)$ vertices of Eqs. (48) through (51), which yield Eq. (68) with Eqs. (69) and (70).

Perhaps the approach taken here can be extended without too much difficulty to calculations beyond NLO. The integrals associated with two-loop contributions will differ from those entering tadpole graphs, but in principle one can construct them from the propagator (Eq. (29)) and the generic forms of loops with (for example) $\mathscr{O}\left(\phi^{6}\right)$ vertex classes from the LO Lagrangian.

\section{Mass vertices}

We begin by considering the vertices in Eq. (47):

$$
-\frac{\mu}{48 f^{2}} \tau_{a b c d} m_{i} \phi_{i j}^{a} \phi_{j k}^{b} \phi_{k l}^{c} \phi_{l i}^{d}
$$

The corresponding tadpole graphs are the sum of all complete contractions with the external fields $\phi_{x y}^{t}$ and $\phi_{y x}^{t}$. Contractions between a field in the vertex and an external field vanish unless the flavor and taste indices match, in which case they contribute factors of unity to amputated diagrams:

$$
\phi_{x y}^{t} \phi_{i j}^{a} \doteq \delta_{x j} \delta_{i y} \delta^{t a}
$$

The remaining fields contract together to give the propagator of Eq. (29) in the loop, and integrating over the loop momentum gives the integral of Eq. (61):

$$
\begin{aligned}
\hat{\phi}_{i j}^{a} \phi_{k l}^{b} & =\delta^{a b}\left(\delta_{i l} \delta_{j k} \frac{1}{q^{2}+\frac{1}{2}\left(I_{a}+J_{a}\right)}+\delta_{i j} \delta_{k l} D_{i l}^{a}\right) \\
& \rightarrow \delta^{a b} K_{i j, k l}^{a} \text { no sum }
\end{aligned}
$$

There are six ways to contract the vertex fields and external fields while maintaining the order of the latter, or equivalently, while maintaining the order of $x$ and $y$. Suppressing the common factors of the coupling $\mu /\left(48 f^{2}\right)$, taste factor $\tau_{a b c d}$, and mass $m_{i}$, we have

$$
\begin{gathered}
\delta_{x j, i y, y k, j x}^{t a, t b, c d} K_{k l, l i}^{c}+\delta_{x j, i y, y l, k x}^{t a, t c, b d} K_{j k, l i}^{b} \\
+\delta_{x j, i y, y i, l x}^{t a, t d, b c} K_{j k, k l}^{b}+\delta_{x k, j y, y l, k x}^{t b, t c, a d} K_{i j, l i}^{a} \\
+\delta_{x k, j y, y i, l x}^{t b, t d, a c} K_{i j, k l}^{a}+\delta_{x l, k y, y i, l x}^{t c, t d, a b} K_{i j, j k}^{a} \\
+(x \leftrightarrow y)
\end{gathered}
$$

where $\delta$ is simply a product of Kronecker deltas:

$$
\delta_{i j, k l, m n, p q}^{a b, c d, e f} \equiv \delta_{i j} \delta_{k l} \delta_{m n} \delta_{p q} \delta^{a b} \delta^{c d} \delta^{e f} .
$$

Restoring the taste factor $\tau_{a b c d}$ and quark mass $m_{i}$ and summing over the flavor and taste indices gives

$$
\begin{gathered}
\tau_{t t c c} m_{y} K_{y i, i y}^{c}+\tau_{t b t b} m_{y} K_{x x, y y}^{b} \\
+\tau_{t b b t} m_{y} K_{x i, i x}^{b}+\tau_{a t t a} m_{i} K_{y i, i y}^{a} \\
+\tau_{a t a t} m_{y} K_{x x, y y}^{a}+\tau_{a a t t} m_{y} K_{y i, i y}^{a} \\
+(x \leftrightarrow y)
\end{gathered}
$$

where we used the symmetry of the propagator under interchange of the fields and relabeled dummy flavor indices. The taste matrices $T^{a}$ all commute or anticommute with one another. Defining $\theta^{a b}$ such that

$$
T^{a} T^{b}=\theta^{a b} T^{b} T^{a} \quad \forall \quad a, b
$$

and noting

$$
\left(T^{a}\right)^{2}=\xi_{I} \quad \forall \quad a,
$$

Eq. (C5) becomes

$$
\begin{aligned}
4 \sum_{a} & {\left[\left(2 m_{x}+m_{y}+m_{i}\right) K_{x i, i x}^{a}\right.} \\
+ & +\left(2 m_{y}+m_{x}+m_{i}\right) K_{y i, i y}^{a} \\
& \left.+2 \theta^{a t}\left(m_{x}+m_{y}\right) K_{x x, y y}^{a}\right] .
\end{aligned}
$$

Restoring the coupling gives the desired result, Eq. (66).

Contributions from loops with kinetic energy, mass, and hairpin vertices (respectively Eqs. (63), (67), and (77)) cancel against the term with $\theta^{\text {at }}$ in Eq. (66), and evaluations of the sums over $\theta^{\text {at }}$ (within irreps of $S O(4)_{T}$ ) are not needed to arrive at the coefficient of the mass term in the result, Eq. (97). In general such cancellations do not occur. The needed sums are collected in Appendix D. 


\section{Potential $a^{2} \mathscr{U}$ vertices}

We begin by noting that the flavor structure of the vertices from $a^{2} \mathscr{U}$ is identical to the flavor structure of the mass vertices (Eq. (C1)):

$$
\begin{aligned}
& -\frac{a^{2} C_{1}}{12 f^{4}}\left(\tau_{a b c d}+3 \tau_{5 a b 5 c d}-4 \tau_{5 a 5 b c d}\right) \phi_{i j}^{a} \phi_{j k}^{b} \phi_{k l}^{c} \phi_{l i}^{d} \quad(\mathrm{C} 9) \\
& -\frac{a^{2} C_{6}}{12 f^{4}}\left(\tau_{a b c d}+3 \tau_{\mu \nu, a b, \mu \nu, c d}-4 \tau_{\mu \nu, a, \mu \nu, b c d}\right) \phi_{i j}^{a} \phi_{j k}^{b} \phi_{k l}^{c} \phi_{l i}^{d} \\
& -\frac{a^{2} C_{3}}{12 f^{4}}\left(\tau_{a b c d}+3 \tau_{\nu a b \nu c d}+4 \tau_{\nu a \nu b c d}\right) \phi_{i j}^{a} \phi_{j k}^{b} \phi_{k l}^{c} \phi_{l i}^{d}(\mathrm{C} 11) \\
& -\frac{a^{2} C_{4}}{12 f^{4}}\left(\tau_{a b c d}+3 \tau_{\nu 5, a b, \nu 5, c d}+4 \tau_{\nu 5, a, \nu 5, b c d}\right) \phi_{i j}^{a} \phi_{j k}^{b} \phi_{k l}^{c} \phi_{l i}^{d} .
\end{aligned}
$$

Only the overall normalizations (couplings) and taste factors differ from those in Eq. (C1). Therefore the same contractions and corresponding products of Kronecker deltas and integrals that appear in Eq. (C3) enter the calculation, and we can obtain the loops for each of the vertex classes in Eqs. (C9) through (C12) from Eqs. (C3) and (C5) by taking $m_{i} \rightarrow 1$ and replacing $\tau_{a b c d}$ in Eq. (C5) with the appropriate linear combinations of traces from Eqs. C9 through (C12).

Noting that the taste factors in Eqs. (C9) through (C12) all have the same form, viz.

$$
\tau_{a b c d}+3 \tau_{s a b s c d}-4 \theta^{5 s} \tau_{s a s b c d}
$$

where

$$
s= \begin{cases}5 & \text { for vertices } \propto C_{1} \\ \mu \nu & \text { for vertices } \propto C_{6} \\ \nu & \text { for vertices } \propto C_{3} \\ \nu 5 & \text { for vertices } \propto C_{4}\end{cases}
$$

and recalling Eqs. (C6) and (C7), we find the taste factors in Eqs. (C9) through (C12) are

$$
\begin{gathered}
\tau_{t t c c}+3 \tau_{s t t s c c}-4 \theta^{5 s} \tau_{\text {ststcc }}=16\left(1-\theta^{5 s} \theta^{s t}\right) \\
\tau_{t b t b}+3 \tau_{\text {stbstb }}-4 \theta^{5 s} \tau_{\text {stsbtb }} \\
=4 \theta^{b t}\left(1+3 \theta^{b s} \theta^{s t}-4 \theta^{5 s} \theta^{s t}\right) \\
\tau_{\text {tbbt }}+3 \tau_{\text {stbsbt }}-4 \theta^{5 s} \tau_{\text {stsbbt }}=4\left(1+3 \theta^{b s} \theta^{s t}-4 \theta^{5 s} \theta^{s t}\right) \\
\tau_{\text {atta }}+3 \tau_{\text {satsta }}-4 \theta^{5 s} \tau_{\text {sastta }}=4\left(1+3 \theta^{a s} \theta^{s t}-4 \theta^{5 s} \theta^{s a}\right) \\
\tau_{\text {atat }}+3 \tau_{\text {satsat }}-4 \theta^{5 s} \tau_{\text {sastat }} \\
=4 \theta^{a t}\left(1+3 \theta^{\text {as }} \theta^{\text {st }}-4 \theta^{5 s} \theta^{s a}\right) \\
\tau_{\text {aatt }}+3 \tau_{\text {saastt }}-4 \theta^{5 s} \tau_{\text {sasatt }}=16\left(1-\theta^{5 s} \theta^{s a}\right) .
\end{gathered}
$$

Replacing the taste factors in Eq. (C5) with these (and taking $m_{i} \rightarrow 1$ there) leads directly to Eq. (68).

\section{Appendix D: Coefficient calculations}

Here we detail one way to calculate the coefficients in Tables I, II, and III. The coefficients defined in Eqs. (69) and (70) can be computed similarly. As a by-product of our calculation, we obtain the intermediate results of Tables $\mathrm{V}$ and $\mathrm{VI}$, which we calculated twice: Once using the (anti)commutation relations of the generators to count the positive and negative terms in each sum, and once using the relation $\theta^{a b}=\tau_{a b a b} / 4$ and a computer. We have also checked Table $\nabla(\mathrm{VI})$ implicitly, using an independent accounting scheme to arrive at the coefficients $\Delta_{B F}^{c o n}\left(\Delta_{B F}^{\text {disc }}\right)$ given in Table 【 (III).

The coefficients $\delta_{B F}^{c o n}$ are defined in Eq. (89):

$$
\begin{aligned}
\delta_{B F}^{c o n}= & \frac{1}{32} \sum_{a \in B} \sum_{b \in V, A} \omega_{b} \tau_{a b t} \tau_{a b t}\left(1+\theta^{a b}\right) \\
=\frac{1}{32} \sum_{\mu, a \in B} & {\left[\omega_{V}\left(\tau_{a \mu t}\right)^{2}\left(1+\theta^{a \mu}\right)\right.} \\
& \left.+\omega_{A}\left(\tau_{a, \mu 5, t}\right)^{2}\left(1+\theta^{a, \mu 5}\right)\right],
\end{aligned}
$$

and we note

$$
1+\theta^{a, \mu(5)}= \begin{cases}2 & \text { if }\left[T^{a}, \xi_{\mu(5)}\right]=0 \\ 0 & \text { if }\left\{T^{a}, \xi_{\mu(5)}\right\}=0\end{cases}
$$

so that

$$
\delta_{P F}^{c o n}=0 \quad \forall F
$$

and

$$
\begin{aligned}
\delta_{I F}^{c o n} & =\frac{1}{16} \sum_{\mu}\left[\omega_{V}\left(\tau_{\mu t}\right)^{2}+\omega_{A}\left(\tau_{\mu 5, t}\right)^{2}\right] \\
& =\sum_{\mu}\left[\omega_{V} \delta^{\mu t}+\omega_{A} \delta^{\mu 5, t}\right] \\
\delta_{I F}^{c o n} & = \begin{cases}\omega_{V} & \text { if } F=V \\
\omega_{A} & \text { if } F=A \\
0 & \text { otherwise. }\end{cases}
\end{aligned}
$$

Similarly, for $B=V$ we have

$$
\begin{aligned}
& \delta_{V F}^{c o n}=\frac{1}{32} \sum_{\mu, \nu}\left[\omega_{V}\left(\tau_{\nu \mu t}\right)^{2}\left(1+\theta^{\nu \mu}\right)\right. \\
& \left.+\omega_{A}\left(\tau_{\nu, \mu 5, t}\right)^{2}\left(1+\theta^{\nu, \mu 5}\right)\right] \\
& =\frac{1}{16} \sum_{\mu} \omega_{V}\left(\tau_{t}\right)^{2}+\frac{1}{8} \sum_{\mu<\nu} \omega_{A}\left(\tau_{\nu, \mu 5, t}\right)^{2} \\
& \delta_{V F}^{c o n}= \begin{cases}4 \omega_{V} & \text { if } F=I \\
2 \omega_{A} & \text { if } F=T \\
0 & \text { otherwise }\end{cases}
\end{aligned}
$$


and for $B=A$, we have

$$
\begin{aligned}
& \delta_{A F}^{c o n}=\frac{1}{32} \sum_{\mu, \nu}\left[\omega_{V}\left(\tau_{\nu 5, \mu t}\right)^{2}\left(1+\theta^{\nu 5, \mu}\right)\right. \\
& \left.+\omega_{A}\left(\tau_{\nu 5, \mu 5, t}\right)^{2}\left(1+\theta^{\nu 5, \mu 5}\right)\right] \\
& =\frac{1}{32} \sum_{\mu, \nu}\left[\omega_{V}\left(\tau_{\nu, \mu 5, t}\right)^{2}\left(1+\theta^{\mu 5, \nu}\right)\right. \\
& \left.+\omega_{A}\left(\tau_{\nu \mu t}\right)^{2}\left(1+\theta^{\nu \mu}\right)\right] \\
& \delta_{A F}^{c o n}= \begin{cases}2 \omega_{V} & \text { if } F=T \\
4 \omega_{A} & \text { if } F=I \\
0 & \text { otherwise. }\end{cases}
\end{aligned}
$$

Finally, for $B=T$ we have

$$
\begin{aligned}
& \delta_{T F}^{c o n}= \frac{1}{32} \sum_{\mu, \rho<\lambda}\left[\omega_{V}\left(\tau_{\rho \lambda, \mu t}\right)^{2}\left(1+\theta^{\rho \lambda, \mu}\right)\right. \\
&\left.+\omega_{A}\left(\tau_{\rho \lambda, \mu 5, t}\right)^{2}\left(1+\theta^{\rho \lambda, \mu 5}\right)\right] \\
&= \frac{1}{16} \sum_{\substack{\rho<\lambda \\
\mu \neq \rho, \lambda}}\left[\omega_{V}\left(\tau_{\rho \lambda, \mu t}\right)^{2}+\omega_{A}\left(\tau_{\rho \lambda, \mu 5, t}\right)^{2}\right] \\
& \delta_{T F}^{c o n}= \begin{cases}3 \omega_{V} & \text { if } F=A \\
3 \omega_{A} & \text { if } F=V \\
0 & \text { otherwise. }\end{cases}
\end{aligned}
$$

These results are straightforwardly obtained by substituting for $t$ and counting the number of nonzero terms in the sums. For evaluating the traces, the (anti)commutation of the generators $T^{a}$, the fact that $\left(T^{a}\right)^{2}=\xi_{I}$, the orthogonality relations $\operatorname{Tr}\left(T^{a} T^{b}\right)=4 \delta^{a b}$, and the traces over products of Euclidean gamma matrices $\gamma_{\mu}$ are useful.

TABLE V. Sums for evaluating the coefficients $\Delta_{B F}^{c o n}$. All the sums required for the coefficients $\Delta_{B F}^{c o n}$ can be obtained by repeated use of the results in the first three lines.

\begin{tabular}{r|rrrrr}
\hline \hline$t \in$ & $P$ & $I$ & $V$ & $A$ & $T$ \\
\hline$\sum_{\mu} \theta^{\mu t}$ & -4 & 4 & -2 & 2 & 0 \\
$\sum_{\mu} \theta^{\mu 5, t}$ & -4 & 4 & 2 & -2 & 0 \\
$\sum_{\mu<\nu} \theta^{\mu \nu, t}$ & 6 & 6 & 0 & 0 & -2 \\
$\sum_{\rho, \mu<\nu} \theta^{\mu \nu, \rho} \theta^{\mu \nu, t}$ & 0 & 0 & 0 & 0 & 0 \\
$\sum_{\rho, \mu} \theta^{\mu \rho} \theta^{\mu t}$ & 8 & -8 & 4 & -4 & 0 \\
$\sum_{\rho, \mu} \theta^{\mu \rho} \theta^{\mu 5, t}$ & 8 & -8 & -4 & 4 & 0 \\
$\sum_{\rho<\lambda, \mu<\nu} \theta^{\mu \nu, \rho \lambda} \theta^{\mu \nu, t}$ & -12 & -12 & 0 & 0 & 4 \\
$\sum_{\rho<\lambda, \mu} \theta^{\mu, \rho \lambda} \theta^{\mu t}$ & 0 & 0 & 0 & 0 & 0 \\
$\sum_{\rho<\lambda, \mu} \theta^{\mu, \rho \lambda} \theta^{\mu 5, t}$ & 0 & 0 & 0 & 0 & 0 \\
\hline \hline
\end{tabular}

The coefficients of Table II are defined in Eq. (90):

$$
\Delta_{B F}^{c o n}=\sum_{a \in B}\left(\Delta_{a t}-\left(\Delta_{t}+\Delta_{a}\right)\right) .
$$

Substituting for the coefficients $\Delta_{a t}$ and taste splittings using Eqs. (69) and (86) gives

$$
\begin{aligned}
\Delta_{B F}^{c o n} & =\frac{24}{f^{2}} \sum_{\substack{a \in B \\
b \neq I}} C_{b}\left(1+\theta^{a b} \theta^{b t}-\theta^{5 b} \theta^{b t}-\theta^{a b} \theta^{b 5}\right) \\
=\frac{24}{f^{2}} & \sum_{a \in B}\left(C_{1}\left(1+\theta^{5 a} \theta^{5 t}-\theta^{5 t}-\theta^{5 a}\right)\right. \\
& +\sum_{\mu<\nu} C_{6}\left(1+\theta^{\mu \nu, a} \theta^{\mu \nu, t}-\theta^{\mu \nu, t}-\theta^{\mu \nu, a}\right) \\
& +\sum_{\mu} C_{3}\left(1+\theta^{\mu a} \theta^{\mu t}+\theta^{\mu t}+\theta^{\mu a}\right) \\
& \left.+\sum_{\mu} C_{4}\left(1+\theta^{\mu 5, a} \theta^{\mu 5, t}+\theta^{\mu 5, t}+\theta^{\mu 5, a}\right)\right) .
\end{aligned}
$$

Writing out the sum over each irrep $B$ gives

$$
\begin{aligned}
\Delta_{P F}^{c o n} & =0 \\
\Delta_{I F}^{c o n} & =\frac{48}{f^{2}} \sum_{\mu}\left(C_{3}\left(1+\theta^{\mu t}\right)+C_{4}\left(1+\theta^{\mu 5, t}\right)\right) \\
\Delta_{V F}^{c o n} & =\frac{24}{f^{2}} \sum_{\rho}\left(2 C_{1}\left(1-\theta^{5 t}\right)\right. \\
& +\sum_{\mu<\nu} C_{6}\left(1+\theta^{\mu \nu, \rho} \theta^{\mu \nu, t}-\theta^{\mu \nu, t}-\theta^{\mu \nu, \rho}\right) \\
& +\sum_{\mu} C_{3}\left(1+\theta^{\mu \rho} \theta^{\mu t}+\theta^{\mu t}+\theta^{\mu \rho}\right) \\
& \left.+\sum_{\mu} C_{4}\left(1-\theta^{\mu \rho} \theta^{\mu 5, t}+\theta^{\mu 5, t}-\theta^{\mu \rho}\right)\right) \\
\Delta_{A F}^{c o n} & =\frac{24}{f^{2}} \sum_{\rho}\left(2 C_{1}\left(1-\theta^{5 t}\right)\right. \\
& +\sum_{\mu<\nu} C_{6}\left(1+\theta^{\mu \nu, \rho} \theta^{\mu \nu, t}-\theta^{\mu \nu, t}-\theta^{\mu \nu, \rho}\right) \\
& \left.+\sum_{\mu} C_{4}\left(1+\theta^{\mu, \rho \lambda} \theta^{\mu 5, t}+\theta^{\mu 5, t}+\theta^{\mu, \rho \lambda}\right)\right) \\
& +\sum_{\mu} C_{3}\left(1-\theta^{\mu \rho} \theta^{\mu t}+\theta^{\mu t}-\theta^{\mu \rho}\right) \\
& \left.+\sum_{\mu} C_{4}\left(1+\theta^{\mu \rho} \theta^{\mu 5, t}+\theta^{\mu 5, t}+\theta^{\mu \rho}\right)\right) \\
& =\frac{24}{f^{2}} \sum_{\rho<\lambda}\left(\sum_{\mu<\nu} C_{6} \times\right.
\end{aligned}
$$

Inspecting Eqs. (D29) through (D41), we note the requisite sums. Their values are given in Table $\mathrm{V}$. Using Table V to evaluate Eqs. (D29) through (D41) yields the 
results of Table II For $F=P(t=5)$, all the coefficients explicitly vanish, as they must.

TABLE VI. Sums (in addition to those in Table V for evaluating the coefficients $\Delta_{B F}^{\text {disc }}$. The last three lines can be obtained from the first two, since $\theta^{\mu 5, t}=\theta^{5 t} \theta^{\mu t}$.

\begin{tabular}{r|rrrrr}
\hline \hline$t \in$ & $P$ & $I$ & $V$ & $A$ & $T$ \\
\hline$\sum_{\rho, \mu<\nu} \theta^{\rho t} \theta^{\mu \nu, \rho} \theta^{\mu \nu, t}$ & 0 & 0 & 12 & -12 & 0 \\
$\sum_{\rho, \mu} \theta^{\rho t} \theta^{\mu \rho} \theta^{\mu t}$ & -8 & -8 & 4 & 4 & 8 \\
$\sum_{\rho<\lambda, \mu<\nu} \theta^{\rho \lambda, t} \theta^{\mu \nu, \rho \lambda} \theta^{\mu \nu, t}$ & -12 & -12 & 0 & 0 & 20 \\
$\sum_{\rho, \mu} \theta^{\rho t} \theta^{\mu \rho} \theta^{\mu 5, t}$ & -8 & -8 & -4 & -4 & 8 \\
$\sum_{\rho, \mu<\nu} \theta^{\rho 5, t} \theta^{\mu \nu, \rho} \theta^{\mu \nu, t}$ & 0 & 0 & -12 & 12 & 0 \\
$\sum_{\rho, \mu} \theta^{\rho 5, t} \theta^{\mu \rho} \theta^{\mu 5, t}$ & -8 & -8 & 4 & 4 & 8 \\
\hline \hline
\end{tabular}

The coefficients of Table III are defined in Eq. (98):

$$
\Delta_{B F}^{d i s c}=\sum_{a \in B}\left(\Delta_{a t}^{\prime}+\theta^{a t} \Delta_{t}+\left(1+\rho^{a t} / 2\right) \Delta_{a}\right)
$$

Substituting for the coefficients $\Delta_{a t}^{\prime}$, taste splittings, and coefficients $\rho^{a t}$ using Eqs. (70), (86) and (69), and (94) gives, for $B \neq I$,

$$
\Delta_{B F}^{d i s c}=\frac{24}{f^{2}} \sum_{\substack{a \in B \\ b \neq I}} C_{b}\left(-1+\theta^{a t}\left(\theta^{a b} \theta^{b t}-\theta^{5 b} \theta^{b t}\right)+\theta^{a b} \theta^{b 5}\right),
$$

and for $B=I$,

$$
\Delta_{I F}^{d i s c}=\frac{24}{f^{2}} \sum_{b \neq I} C_{b}\left(1+\theta^{b t}-\theta^{5 b} \theta^{b t}-\theta^{b 5}\right) .
$$

For $B \neq I$, adding and subtracting Eqs. (D43) and (D23) gives

$$
\begin{aligned}
& \Delta_{B I}^{d i s c}=\Delta_{B I}^{c o n}-6 N_{B} \Delta_{B} \quad(B \neq I) \\
& \Delta_{P T}^{d i s c}=\Delta_{P T}^{c o n} \\
& \Delta_{T P}^{d i s c}=\Delta_{T P}^{c o n}-36 \Delta_{T} \\
& \Delta_{P V}^{d i s c}=-\Delta_{P V}^{c o n} \\
& \Delta_{P A}^{d i s c}=-\Delta_{P A}^{c o n} \\
& \Delta_{V P}^{d i s c}=-\Delta_{V P}^{c o n} \\
& \Delta_{A P}^{d i s c}=-\Delta_{A P}^{c o n},
\end{aligned}
$$

while for $B=I$, comparing Eqs. (D44) and (D23) gives

$$
\Delta_{I F}^{\text {disc }}=\Delta_{I F}^{c o n}
$$

Eq. (D45) implies Eqs. (101) and (102); they and Eqs. (D50) and (D51) can be used to cross-check the results in Table III. Eq. (D52) and Table II give the coefficients $\Delta_{I F}^{d i s c}$, while the coefficients $\Delta_{P F}^{d i s c}$ and $\Delta_{T F}^{d i s c}$ do not appear in Eq. (97); the remaining coefficients in Eq. (97) are $\Delta_{V F}^{\text {disc }}$ and $\Delta_{A F}^{\text {disc }}$.

Writing out the sum over $b$ in Eq. (D43) gives

$$
\begin{aligned}
& \Delta_{B F}^{d i s c}=\frac{24}{f^{2}} \sum_{a \in B}\left(C_{1}\left(-1+\theta^{a t}\left(\theta^{5 a} \theta^{5 t}-\theta^{5 t}\right)+\theta^{5 a}\right)\right. \\
& +\sum_{\mu<\nu} C_{6}\left(-1+\theta^{a t}\left(\theta^{\mu \nu, a} \theta^{\mu \nu, t}-\theta^{\mu \nu, t}\right)+\theta^{\mu \nu, a}\right) \\
& +\sum_{\mu} C_{3}\left(-1+\theta^{a t}\left(\theta^{\mu a} \theta^{\mu t}+\theta^{\mu t}\right)-\theta^{\mu a}\right) \\
& \left.+\sum_{\mu} C_{4}\left(-1+\theta^{a t}\left(\theta^{\mu 5, a} \theta^{\mu 5, t}+\theta^{\mu 5, t}\right)-\theta^{\mu 5, a}\right)\right)
\end{aligned}
$$

and writing out the sums over the vector and axial irreps $(B=V$ and $B=A$ ) gives

$$
\begin{aligned}
& \Delta_{V F}^{d i s c}=\frac{24}{f^{2}} \sum_{\rho}\left(2 C_{1}\left(-1-\theta^{\rho t} \theta^{5 t}\right)\right. \\
& +\sum_{\mu<\nu} C_{6}\left(-1+\theta^{\rho t}\left(\theta^{\mu \nu, \rho} \theta^{\mu \nu, t}-\theta^{\mu \nu, t}\right)+\theta^{\mu \nu, \rho}\right) \\
& +\sum_{\mu} C_{3}\left(-1+\theta^{\rho t}\left(\theta^{\mu \rho} \theta^{\mu t}+\theta^{\mu t}\right)-\theta^{\mu \rho}\right) \\
& \left.+\sum_{\mu} C_{4}\left(-1-\theta^{\rho t}\left(\theta^{\mu \rho} \theta^{\mu 5, t}-\theta^{\mu 5, t}\right)+\theta^{\mu \rho}\right)\right) \\
& \Delta_{A F}^{d i s c}=\frac{24}{f^{2}} \sum_{\rho}\left(2 C_{1}\left(-1-\theta^{\rho 5, t} \theta^{5 t}\right)\right. \\
& +\sum_{\mu<\nu} C_{6}\left(-1+\theta^{\rho 5, t}\left(\theta^{\mu \nu, \rho} \theta^{\mu \nu, t}-\theta^{\mu \nu, t}\right)+\theta^{\mu \nu, \rho}\right) \\
& +\sum_{\mu} C_{3}\left(-1-\theta^{\rho 5, t}\left(\theta^{\mu \rho} \theta^{\mu t}-\theta^{\mu t}\right)+\theta^{\mu \rho}\right) \\
& \left.+\sum_{\mu} C_{4}\left(-1+\theta^{\rho 5, t}\left(\theta^{\mu \rho} \theta^{\mu 5, t}+\theta^{\mu 5, t}\right)-\theta^{\mu \rho}\right)\right)
\end{aligned}
$$

Examining Eqs. (D57) through (D64), we note the sums beyond those in Table $\mathrm{V}$ that are needed to evaluate the coefficients $\Delta_{V F}^{d i s c}$ and $\Delta_{A F}^{d i s c}$. The values of these sums are given in Table VI. Using the sums in Tables V] and VI in Eqs. (D57) through (D64) yields the results in Table III. From Eq. (D44) and Eqs. (D53) through (D56), we see that $\Delta_{I P}^{\text {disc }}=\Delta_{V P}^{\text {disc }}=\Delta_{A P}^{\text {disc }}=0$, as necessary for the result in Eq. (97) to reduce properly in the taste Goldstone case. 
[1] J. Gasser and H. Leutwyler, Annals Phys. 158, 142 (1984).

[2] J. Gasser and H. Leutwyler, Nucl.Phys. B250, 465 (1985).

[3] G. Colangelo, S. Durr, A. Juttner, L. Lellouch, H. Leutwyler, et al., Eur.Phys.J. C71, 1695 (2011), 1011.4408 .

[4] A. Bazavov, D. Toussaint, C. Bernard, J. Laiho, C. DeTar, et al., Rev.Mod.Phys. 82, 1349 (2010), 0903.3598.

[5] K. Symanzik, Nucl.Phys. B226, 187 (1983).

[6] K. Symanzik, Nucl.Phys. B226, 205 (1983).

[7] W.-J. Lee and S. R. Sharpe, Phys.Rev. D60, 114503 (1999), hep-lat/9905023.

[8] N. Ishizuka, M. Fukugita, H. Mino, M. Okawa, and A. Ukawa, Nucl.Phys. B411, 875 (1994).

[9] K. Orginos and D. Toussaint (MILC collaboration), Phys.Rev. D59, 014501 (1999), hep-lat/9805009.

[10] C. W. Bernard, T. Burch, K. Orginos, D. Toussaint, T. A. DeGrand, et al., Phys.Rev. D64, 054506 (2001), heplat/0104002.

[11] C. Bernard (MILC Collaboration), Phys.Rev. D65, 054031 (2002), hep-lat/0111051.

[12] C. Aubin and C. Bernard, Phys.Rev. D68, 034014 (2003), hep-lat/0304014.

[13] C. Aubin and C. Bernard, Nucl.Phys.Proc.Suppl. 129, 182 (2004), hep-lat/0308036.

[14] C. Aubin and C. Bernard, Phys.Rev. D68, 074011 (2003), hep-lat/0306026.

[15] C. Aubin et al. (HPQCD Collaboration, MILC Collaboration, UKQCD Collaboration), Phys.Rev. D70, 031504 (2004), hep-lat/0405022.

[16] C. Aubin et al. (MILC Collaboration), Phys.Rev. D70, 114501 (2004), hep-lat/0407028.

[17] C. Aubin et al. (Fermilab Lattice Collaboration, MILC Collaboration, HPQCD Collaboration), Phys.Rev.Lett. 94, 011601 (2005), hep-ph/0408306.

[18] C. Aubin, C. Bernard, C. E. DeTar, M. Di Pierro, E. D. Freeland, et al., Phys.Rev.Lett. 95, 122002 (2005), heplat/0506030.

[19] A. Gray et al. (HPQCD Collaboration), Phys.Rev.Lett. 95, 212001 (2005), hep-lat/0507015.

[20] C. Aubin and T. Blum, PoS LAT2005, 089 (2006), heplat/0509064.

[21] M. Okamoto, PoS LAT2005, 013 (2006), heplat/0510113.

[22] E. Dalgic, A. Gray, M. Wingate, C. T. Davies, G. Lepage, et al., Phys.Rev. D73, 074502 (2006), hep-lat/0601021.

[23] C. Aubin and T. Blum, Phys.Rev. D75, 114502 (2007), hep-lat/0608011.

[24] C. Bernard, C. E. DeTar, M. Di Pierro, A. ElKhadra, R. Evans, et al., Phys.Rev. D79, 014506 (2009), 0808.2519.

[25] J. A. Bailey, C. Bernard, C. E. DeTar, M. Di Pierro, A. El-Khadra, et al., Phys.Rev. D79, 054507 (2009), 0811.3640 .

[26] E. Gamiz, C. T. Davies, G. Lepage, J. Shigemitsu, and M. Wingate (HPQCD Collaboration), Phys.Rev. D80, 014503 (2009), 0902.1815.

[27] T. Bae, Y.-C. Jang, C. Jung, H.-J. Kim, J. Kim, et al., Phys.Rev. D82, 114509 (2010), 1008.5179.

[28] J. Kim, C. Jung, H.-J. Kim, W. Lee, and S. R. Sharpe,
Phys.Rev. D83, 117501 (2011), 1101.2685.

[29] S. R. Sharpe and R. S. Van de Water, Phys.Rev. D71, 114505 (2005), hep-lat/0409018.

[30] P. Damgaard and K. Splittorff, Phys.Rev. D62, 054509 (2000), hep-lat/0003017.

[31] G. C. Donald, C. T. Davies, E. Follana, and A. S. Kronfeld, Phys.Rev. D84, 054504 (2011), 1106.2412.

[32] C. Bernard, M. Golterman, and Y. Shamir, Phys.Rev. D77, 074505 (2008), 0712.2560.

[33] S. R. Sharpe, PoS LAT2006, 022 (2006), heplat/0610094.

[34] C. Bernard, Phys.Rev. D73, 114503 (2006), heplat/0603011.

[35] C. Bernard, M. Golterman, and Y. Shamir, PoS LAT2006, 205 (2006), hep-lat/0610003.

[36] C. Bernard, M. Golterman, and Y. Shamir, PoS LAT2007, 263 (2007), 0709.2180.

[37] D. H. Adams, Nucl.Phys.Proc.Suppl. 140, 148 (2005), hep-lat/0409013.

[38] S. Durr, PoS LAT2005, 021 (2006), hep-lat/0509026.

[39] M. Creutz, PoS LAT2007, 007 (2007), 0708.1295.

[40] A. S. Kronfeld, PoS LAT2007, 016 (2007), 0711.0699.

[41] D. H. Adams, Phys.Rev. D77, 105024 (2008), 0802.3029.

[42] M. Creutz, PoS CONFINEMENT8, 016 (2008), 0810.4526 .

[43] M. Golterman, PoS CONFINEMENT8, 014 (2008), 0812.3110 .

[44] M. Golterman (2009), 0912.4042.

[45] S. Sharpe (2006), hep-lat/0607016.

[46] S. Weinberg, Physica A96, 327 (1979), festschrift honoring Julian Schwinger on his 60th birthday.

[47] S. Scherer, Adv.Nucl.Phys. 27, 277 (2003), to be edited by J.W. Negele and E. Vogt, hep-ph/0210398.

[48] S. R. Sharpe and N. Shoresh, Phys.Rev. D62, 094503 (2000), hep-lat/0006017.

[49] S. R. Sharpe and N. Shoresh, Phys.Rev. D64, 114510 (2001), hep-lat/0108003.

[50] C. W. Bernard and M. F. Golterman, Phys.Rev. D46, 853 (1992), hep-lat/9204007.

[51] C. W. Bernard and M. F. Golterman, Phys.Rev. D49, 486 (1994), hep-lat/9306005.

[52] X. Du, Phys.Rev. D82, 014016 (2010), 0911.2534.

[53] A. Bazavov, C. Bernard, C. DeTar, X. Du, W. Freeman, et al., PoS LATTICE2010, 083 (2010), long author list - awaiting processing, 1011.1792.

[54] A. Bazavov et al. (MILC Collaboration), PoS CD09, 007 (2009), 0910.2966.

[55] A. Bazavov et al. (The MILC Collaboration), PoS LAT2009, 077 (2009), 0911.0472.

[56] T. Bae, D. H. Adams, C. Jung, H.-J. Kim, J. Kim, et al., Phys.Rev. D77, 094508 (2008), 0801.3000.

[57] D. Adams, T. Bae, H.-J. Kim, J. Kim, K. Kim, et al., PoS LATTICE2008, 104 (2008), 0809.1219.

[58] O. Bar, G. Rupak, and N. Shoresh, Phys.Rev. D70, 034508 (2004), hep-lat/0306021.

[59] O. Bar, C. Bernard, G. Rupak, and N. Shoresh, Phys.Rev. D72, 054502 (2005), hep-lat/0503009.

[60] J.-W. Chen, D. O'Connell, and A. Walker-Loud, JHEP 0904, 090 (2009), 0706.0035.

[61] J.-W. Chen, M. Golterman, D. O'Connell, and A. Walker-Loud, Phys.Rev. D79, 117502 (2009), 
0905.2566 